\title{
AFFINE STRUCTURES ON TEICHMÜLLER SPACES AND APPLICATIONS*
}

\author{
XIAOJING CHEN $^{\dagger}$, FENG GUAN ${ }^{\ddagger}$, AND KEFENG LIU§
}

\begin{abstract}
We review our recent results on holomorphic affine structures on the Teichmüller spaces of Calabi-Yau manifolds and their Hodge metric completions, as well as their applications to global Torelli theorems for Calabi-Yau manifolds. As corollaries we show that the extended period map from the completion space is injective into the period domain, and that the completion space is a domain of holomorphy and admits a complete Kähler-Einstein metric.
\end{abstract}

Key words. Affine structure, Teichmuller space, Calabi-Yau manifolds, global Torelli theorem.

AMS subject classifications. 32J25, 14C34, 14C30, 32G20, 32G05.

1. Introduction. A compact projective manifold $M$ of complex dimension $n$ with $n \geq 3$ is called Calabi-Yau in this paper, if it has a trivial canonical bundle and satisfies $H^{i}\left(M, \mathcal{O}_{M}\right)=0$ for $0<i<n$. A polarized and marked Calabi-Yau manifold is a triple consisting of a Calabi-Yau manifold $M$, an ample line bundle $L$ over $M$ and a basis of the integral middle homology group modulo torsion, $H_{n}(M, \mathbb{Z}) /$ Tor.

We will denote by $\mathcal{T}$ the Teichmüller space for the deformations of the complex structure on the polarized and marked Calabi-Yau manifold $M$. Actually the Teichmüller space is precisely the universal cover of the smooth moduli space $\mathcal{Z}_{m}$ with level $m$ structure with $m \geq 3$, which is constructed by Popp, Viehweg, and Szendröi, for example in Section 2 of [20]. The versal family $\mathcal{U} \rightarrow \mathcal{T}$ of the polarized and marked Calabi-Yau manifolds is the pull-back of the versal family $\mathcal{X}_{\mathcal{Z}_{m}} \rightarrow \mathcal{Z}_{m}$, which is also introduced in [20]. Therefore $\mathcal{T}$ is a connected and simply connected smooth complex manifold with $\operatorname{dim}_{\mathbb{C}} \mathcal{T}=h^{n-1,1}(M)=N$, where $h^{n-1,1}(M)=\operatorname{dim}_{\mathbb{C}} H^{n-1,1}(M)$.

Let $D$ be the period domain of polarized Hodge structures of the $n$-th primitive cohomology of $M$. The period map $\Phi: \mathcal{T} \rightarrow D$ is defined by assigning to each point in $\mathcal{T}$ the Hodge structure of the corresponding fiber. Let us denote the period map on the smooth moduli space by $\Phi_{\mathcal{Z}_{m}}: \mathcal{Z}_{m} \rightarrow D / \Gamma$, where $\Gamma$ denotes the global monodromy group which acts properly and discontinuously on $D$. Denote by $\pi_{m}: \mathcal{T} \rightarrow \mathcal{Z}_{m}$ the universal covering map. Then $\Phi: \mathcal{T} \rightarrow D$ is the lifting of $\Phi_{\mathcal{Z}_{m}} \circ \pi_{m}$. There is Hodge metric $h$ on $D$, which is a complete homogeneous metric and is studied in [5]. By local Torelli theorem of Calabi-Yau manifolds, both $\Phi_{\mathcal{Z}_{m}}$ and $\Phi$ are locally injective. Thus the pull-backs of $h$ on $\mathcal{Z}_{m}$ and $\mathcal{T}$ are both well-defined Kähler metrics, and they are still called Hodge metrics.

In this paper, one of our essential constructions is the global holomorphic affine structure on the Teichmüller space, which can be outlined as follows: fix a base point $p \in \mathcal{T}$ with its Hodge structure $\left\{H_{p}^{k, n-k}\right\}_{k=0}^{n}$ as the reference Hodge structure. With this fixed base point $\Phi(p) \in D$, we identify the unipotent subgroup $N_{+}$with its orbit in $\check{D}$ (see Section 3.1 and Remark 3.1) and define $\check{\mathcal{T}}=\Phi^{-1}\left(N_{+}\right) \subseteq \mathcal{T}$. We first show that

\footnotetext{
*Received April 4, 2013; accepted for publication August 15, 2013.

$\dagger$ Department of Mathematics, University of California at Los Angeles, Los Angeles, CA 900951555, USA (xjchen@math.ucla.edu).

${ }^{\ddagger}$ Department of Mathematics, University of California at Los Angeles, Los Angeles, CA 900951555, USA (fguan@math.ucla.edu).

$\S$ Center of Mathematical Sciences, Zhejiang University, Hangzhou, Zhejiang 310027, China; Department of Mathematics, University of California at Los Angeles, Los Angeles, CA 90095-1555, USA(liu@math.ucla.edu; liu@cms.zju.edu.cn).
} 
$\Phi: \check{\mathcal{T}} \rightarrow N_{+} \cap D$ is a bounded map with respect to the Euclidean metric on $N_{+}$, and that $\mathcal{T} \backslash \check{\mathcal{T}}$ is an analytic subvariety. Then by applying Riemann extension theorem, we conclude that $\Phi(\mathcal{T}) \subseteq N_{+} \cap D$. Using this property, we then show $\Phi$ induces a global holomorphic map $\tau: \mathcal{T} \rightarrow \mathbb{C}^{N}$, which actually gives a local coordinate map around each point in $\mathcal{T}$ by using local Torelli theorem for Calabi-Yau manifolds. Thus $\tau: \mathcal{T} \rightarrow \mathbb{C}^{N}$ induces a global holomorphic affine structure on $\mathcal{T}$. It is not hard to see that $\tau=P \circ \Phi: \mathcal{T} \rightarrow \mathbb{C}^{N}$ is a composition map with $P: N_{+} \rightarrow \mathbb{C}^{N} \simeq H_{p}^{n-1,1}$ a natural projection map into a subspace, where $N_{+} \simeq \mathbb{C}^{d}$ with the fixed base point $p \in \mathcal{T}$.

Let $\mathcal{Z}_{m}^{H}$ be the Hodge metric completion of the smooth moduli space $\mathcal{Z}_{m}$ and let $\mathcal{T}_{m}^{H}$ be the universal cover of $\mathcal{Z}_{m}^{H}$ with the universal covering map $\pi_{m}^{H}: \mathcal{T}_{m}^{H} \rightarrow \mathcal{Z}_{m}^{H}$. It is easy to see that $\mathcal{Z}_{m}^{H}$ is a connected and complete smooth complex manifold, and thus $\mathcal{T}_{m}^{H}$ is a connected and simply connected complete smooth complex manifold. We also obtain the following commutative diagram:

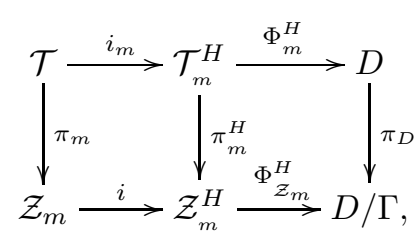

where $\Phi_{\mathcal{Z}_{m}}^{H}$ is the natural extension map of the period map $\Phi_{\mathcal{Z}_{m}}: \mathcal{Z}_{m} \rightarrow D / \Gamma, i$ is the inclusion map, $i_{m}$ is a lifting of $i \circ \pi_{m}$, and $\Phi_{m}^{H}$ is a lifting of $\Phi_{\mathcal{Z}_{m}}^{H} \circ \pi_{m}^{H}$. It is not hard to see that $\Phi_{m}^{H}$ is actually a bounded holomorphic map from $\mathcal{T}_{m}^{H}$ to $N_{+} \cap D$.

We first prove that the complete complex manifold $\mathcal{T}_{m}^{H}$ is a complex affine manifold and the extended period map $\Phi_{m}^{H}: \mathcal{T}_{m}^{H} \rightarrow D$ is injective. This is carried out by crucially using the holomorphic affine structure on $\mathcal{T}$. Then we show that $\mathcal{T}_{m}^{H}$ is independent of the choice of $m$. More precisely we have that, for any $m, m^{\prime} \geq 3$, the complex manifolds $\mathcal{T}_{m}^{H}$ and $\mathcal{T}_{m^{\prime}}^{H}$ are biholomorphic to each other. This allows us to define the complete complex manifold $\mathcal{T}^{H}$ by $\mathcal{T}^{H}=\mathcal{T}_{m}^{H}$, the holomorphic map $i_{\mathcal{T}}: \mathcal{T} \rightarrow \mathcal{T}^{H}$ by $i_{\mathcal{T}}=i_{m}$, and the holomorphic map $\Phi^{H}: \mathcal{T}^{H} \rightarrow D$ by $\Phi^{H}=\Phi_{m}^{H}$ for any $m \geq 3$. With these new notations, we have the commutative diagram

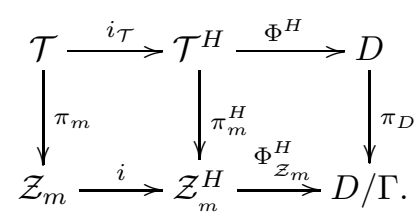

The main result of this paper is the following.

THEOREM 1.1. The complete complex affine manifold $\mathcal{T}^{H}$ is the completion space of $\mathcal{T}$ with respect to the Hodge metric, and it is a bounded domain in $\mathbb{C}^{N}$. Moreover, the extended period map $\Phi^{H}: \mathcal{T}^{H} \rightarrow N_{+} \cap D$ is a holomorphic injection.

One technical difficulty of our arguments is to show directly that $\mathcal{T}^{H}$ is indeed the Hodge metric completion space of $\mathcal{T}$, and for this it is sufficient to show that $i_{\mathcal{T}}: \mathcal{T} \rightarrow \mathcal{T}^{H}$ is an embedding. To overcome this difficulty we have to go through the space $\mathcal{T}_{m}^{H}$. In fact, defining $\mathcal{T}_{m}$ to be $i_{m}(\mathcal{T})$ and denoting it by $\mathcal{T}_{0}$, since $\mathcal{T}_{m}$ is independent of choice of $m$, we have $\mathcal{T}_{0}=i_{\mathcal{T}}(\mathcal{T}) \subseteq \mathcal{T}^{H}$. It is not hard to show that 
$i_{\mathcal{T}}: \mathcal{T} \rightarrow \mathcal{T}_{0}$ is a covering map. Moreover, we prove that $i_{\mathcal{T}}: \mathcal{T} \rightarrow \mathcal{T}_{0}$ is actually one-to-one by showing that the fundamental group of $\mathcal{T}_{0}$ is trivial. Therefore, we can conclude that $i_{\mathcal{T}}: \mathcal{T} \rightarrow \mathcal{T}^{H}$ is an embedding. Here the markings of the Calabi-Yau manifolds and the simply connectedness of $\mathcal{T}$ come into play substantially.

As a direct corollary of this theorem, we easily deduce that the period map $\Phi=$ $\Phi^{H} \circ i_{\mathcal{T}}: \mathcal{T} \rightarrow D$ is also injective since it is a composition of two injective maps. This is the global Torelli theorem for the period map from the Teichmüller space to the period domain. In case that the period domain $D$ is Hermitian symmetric and that it has the same dimension as $\mathcal{T}$, the above theorem implies that the extended period map $\Phi^{H}$ is biholomorphic, in particular it is surjective. As another important result of this paper, we prove the following theorem.

THEOREM 1.2. The completion space $\mathcal{T}^{H}$ is a domain of holomorphy in $\mathbb{C}^{N}$; thus there exists a complete Kähler-Einstein metric on $\mathcal{T}^{H}$.

To prove this theorem, we construct a plurisubharmonic exhaustion function on $\mathcal{T}^{H}$ by using Proposition 5.8, the completeness of $\mathcal{T}^{H}$, and the injectivity of $\Phi^{H}$. This shows that $\mathcal{T}^{H}$ is a domain of holomorphy in $\mathbb{C}^{N}$. The existence of the Kähler-Einstein metric follows directly from a theorem of Mok-Yau in [11].

This paper is organized as follows. In Section 2, we review the definition of the period domain of polarized Hodge structures, the construction of the Teichmüller space of polarized and marked Calabi-Yau manifolds, the definition of the period map and the Hodge metrics on the moduli space and the Teichmüller space respectively. In Section 3, we show that the image of the period map is in $N_{+} \cap D$ and we construct a holomorphic affine structure on the Teichmüller space. In Section 4, we prove that there exists a global holomorphic affine structure on $\mathcal{T}_{m}^{H}$ and that the map $\Phi_{m}^{H}: \mathcal{T}_{m}^{H} \rightarrow D$ is an injective map. In Section 5, we first show that $\mathcal{T}_{m}^{H}$ does not depend on $m$ for all $m \geq 3$. Then we define the completion space $\mathcal{T}^{m}$ and the extended period map $\Phi^{H}$. As our main results, we show that $\mathcal{T}^{H}$ is a complex affine manifold, and it is the Hodge metric completion space of $\mathcal{T}$. Moreover, we show that $\Phi^{H}$ is a holomorphic injection. Therefore, the global Torelli theorem for CalabiYau manifolds from the Teichmüller space follows directly. Finally we prove $\mathcal{T}^{H}$ is a domain of holomorphy in $\mathbb{C}^{N}$, and thus it admits a complete Kähler-Einstein metric.

In this paper we only outline the main ideas of our proofs. For details of the arguments we refer the reader to [1]. We remark that the same results hold for projective manifolds of Calabi-Yau type, which is another interesting class of projective Fano manifolds. These results are carried out in [2].

2. Teichmüller space and the period map of Calabi-Yau manifolds. In Section 2.1, we recall the definition and some basic properties of the period domain. In Section 2.2, we discuss the construction of the Teichmülller space of Calabi-Yau manifolds based on the works of Popp [15], Viehweg [23] and Szendröi [20] on the moduli spaces of Calabi-Yau manifolds. In Section 2.3, we define the period map from the Teichmüller space to the period domain. We also describe the Hodge metrics on the moduli space and the Teichmüller space, respectively. We remark that most of the results in this section are standard and can be found from the literature in the subjects. We collect them here for the reader's convenience.

2.1. Period domain of polarized Hodge structures. In this section, we the construction of the period domain of polarized Hodge structures. We refer the reader to Section 3 of [16] for more details. 
A pair $(M, L)$ consisting of a Calabi-Yau manifold $M$ of complex dimension $n$ and an ample line bundle $L$ over $M$ is called a polarized Calabi-Yau manifold. By abuse of notation, the Chern class of $L$ will also be denoted by $L$ and thus $L \in H^{2}(M, \mathbb{Z})$. Let $\left\{\gamma_{1}, \cdots, \gamma_{h^{n}}\right\}$ be a basis of the integral homology group modulo torsion, $H_{n}(M, \mathbb{Z}) /$ Tor, with $\operatorname{dim} H_{n}(M, \mathbb{Z}) /$ Tor.

DeFinition 2.1. Let the pair $(M, L)$ be a polarized Calabi-Yau manifold, we call the triple $\left(M, L,\left\{\gamma_{1}, \cdots, \gamma_{h^{n}}\right\}\right)$ a polarized and marked Calabi-Yau manifold.

For a polarized and marked Calabi-Yau manifold $M$ with background smooth manifold $X$, we identify the basis of $H_{n}(M, \mathbb{Z}) /$ Tor to a lattice $\Lambda$ as in [20]. This gives us a canonical identification of the middle dimensional de Rahm cohomology of $M$ to that of the background manifold $X$, that is,

$$
H^{n}(M) \cong H^{n}(X)
$$

where the coefficient ring can be $\mathbb{Q}, \mathbb{R}$ or $\mathbb{C}$. Since the polarization $L$ is an integer class, it defines a map

$$
L: H^{n}(X, \mathbb{Q}) \rightarrow H^{n+2}(X, \mathbb{Q})
$$

given by $A \mapsto L \wedge A$ for any $A \in H^{n}(X, \mathbb{Q})$. We denote by $H_{p r}^{n}(X)=\operatorname{ker}(L)$ the primitive cohomology groups where, again, the coefficient ring can be $\mathbb{Q}, \mathbb{R}$ or $\mathbb{C}$. We let $H_{p r}^{k, n-k}(M)=H^{k, n-k}(M) \cap H_{p r}^{n}(M, \mathbb{C})$ and denote its dimension by $h^{k, n-k}$. We have the Hodge decomposition

$$
H_{p r}^{n}(M, \mathbb{C})=H_{p r}^{n, 0}(M) \oplus \cdots \oplus H_{p r}^{0, n}(M) .
$$

It is easy to see that for a polarized Calabi-Yau manifold, since $H^{2}\left(M, \mathcal{O}_{M}\right)=0$, we have

$$
H_{p r}^{n, 0}(M)=H^{n, 0}(M), H_{p r}^{n-1,1}(M)=H^{n-1,1}(M) .
$$

The Poincaré bilinear form $Q$ on $H_{p r}^{n}(X, \mathbb{Q})$ is defined by

$$
Q(u, v)=(-1)^{\frac{n(n-1)}{2}} \int_{X} u \wedge v
$$

for any $d$-closed $n$-forms $u, v$ on $X$. The bilinear form $Q$ is symmetric if $n$ is even and is skew-symmetric if $n$ is odd. Furthermore, $Q$ is nondegenerate and can be extended to $H_{p r}^{n}(X, \mathbb{C})$ bilinearly, and satisfies the Hodge-Riemann relations

$$
\begin{array}{r}
Q\left(H_{p r}^{k, n-k}(M), H_{p r}^{l, n-l}(M)\right)=0 \text { unless } k+l=n, \quad \text { and } \\
(\sqrt{-1})^{2 k-n} Q(v, \bar{v})>0 \text { for } v \in H_{p r}^{k, n-k}(M) \backslash\{0\} .
\end{array}
$$

The Hodge decomposition of $H_{p r}^{n}(M, \mathbb{C})$ in $(2)$ can also be described via the Hodge filtration. Let $f^{k}=\sum_{i=k}^{n} h^{i, n-i}, f^{0}=m$, and

$$
F^{k}=F^{k}(M)=H_{p r}^{n, 0}(M) \oplus \cdots \oplus H_{p r}^{k, n-k}(M)
$$

from which we have the decreasing filtration $H_{p r}^{n}(M, \mathbb{C})=F^{0} \supset \cdots \supset F^{n}$. We know that

$$
\begin{gathered}
\operatorname{dim}_{\mathbb{C}} F^{k}=f^{k} \\
H_{p r}^{n}(X, \mathbb{C})=F^{k} \oplus \overline{F^{n-k+1}}, \quad \text { and } \quad H_{p r}^{k, n-k}(M)=F^{k} \cap \overline{F^{n-k}} .
\end{gathered}
$$


In terms of the Hodge filtration $F^{n} \subset \cdots \subset F^{0}=H_{p r}^{n}(M, \mathbb{C})$, the Hodge-Riemann relations (3) and (4) can be written as

$$
\begin{array}{r}
Q\left(F^{k}, F^{n-k+1}\right)=0, \quad \text { and } \\
Q(C v, \bar{v})>0 \text { if } v \neq 0
\end{array}
$$

where $C$ is the Weil operator given by $C v=(\sqrt{-1})^{2 k-n} v$ for $v \in H_{p r}^{k, n-k}(M)$. The period domain $D$ for polarized Hodge structures with data (5) is the space of all such Hodge filtrations

$$
D=\left\{F^{n} \subset \cdots \subset F^{0}=H_{p r}^{n}(X, \mathbb{C}) \mid(5),(7) \text { and (8) hold }\right\} .
$$

The compact dual $\check{D}$ of $D$ is

$$
\check{D}=\left\{F^{n} \subset \cdots \subset F^{0}=H_{p r}^{n}(X, \mathbb{C}) \mid(5) \text { and }(7) \text { hold }\right\} .
$$

The period domain $D \subseteq \check{D}$ is an open set. We note that the conditions (7) and (8) imply the identities in (6). From the definition of period domain we naturally get the Hodge bundles on $\check{D}$ by associating to each point in $\check{D}$ the vector spaces $\left\{F^{k}\right\}_{k=0}^{n}$ in the Hodge filtration of that point. Without confusion we will also denote by $F^{k}$ the bundle with $F^{k}$ as the fiber for each $0 \leq k \leq n$.

REMARK 2.2. We remark the notation change for the primitive cohomology. As mentioned above that for a polarized Calabi-Yau manifold,

$$
H_{p r}^{n, 0}(M)=H^{n, 0}(M), H_{p r}^{n-1,1}(M)=H^{n-1,1}(M) .
$$

For the reason that we mainly consider these two types of primitive cohomology group of a Calabi-Yau manifold, by abuse of notation, we will simply use $H^{n}(M, \mathbb{C})$ and $H^{k, n-k}(M)$ to denote the primitive cohomology groups $H_{p r}^{n}(M, \mathbb{C})$ and $H_{p r}^{k, n-k}(M)$ respectively. Moreover, we will use cohomology to mean primitive cohomology in the rest of the paper.

2.2. Construction of the Teichmüller space. We first recall the concept of Kuranishi family of compact complex manifolds. We refer to page 8-10 in [17], page 94 in [15] or page 19 in [23] for equivalent definitions and more details. If a complex analytic family $\pi: \mathcal{V} \rightarrow \mathcal{B}$ of compact complex manifolds is complete at each point of $\mathcal{B}$ and versal at the point $0 \in \mathcal{B}$, then the family $\pi: \mathcal{V} \rightarrow \mathcal{B}$ is called the Kuranishi family of the complex manifold $V=\pi^{-1}(0)$. The base space $\mathcal{B}$ is called the Kuranishi space. If the family is complete at each point of a neighbourhood of $0 \in \mathcal{B}$ and versal at 0 , then the family is called a local Kuranishi family at $0 \in \mathcal{B}$. In particular, by definition if a family is versal at each point of $\mathcal{B}$, then it is local Kuranishi at every point of $\mathcal{B}$.

Let $(M, L)$ be a polarized Calabi-Yau manifold. We call a basis of the quotient space $\left(H_{n}(M, \mathbb{Z}) /\right.$ Tor $) / m\left(H_{n}(M, \mathbb{Z}) /\right.$ Tor $)$ a level $m$ structure on the polarized Calabi-Yau manifold with $m \geq 3$. For deformation of polarized Calabi-Yau manifold with level $m$ structure, we have the following theorem, which is a reformulation of Theorem 2.2 in [20], we just take the statement we need in this paper. One can also look at [15] and [23] for more details about the construction of moduli spaces of Calabi-Yau manifolds. 
TheOREM 2.3. Let $m \geq 3$ and $M$ be a polarized Calabi-Yau manifold with level $m$ structure. Then there exists a quasi-projective complex manifold $\mathcal{Z}_{m}$ with a versal family of Calabi-Yau manifolds,

$$
\mathcal{X}_{\mathcal{Z}_{m}} \rightarrow \mathcal{Z}_{m}
$$

containing $M$ as a fiber, and polarized by an ample line bundle $\mathcal{L}_{\mathcal{Z}_{m}}$ on $\mathcal{X}_{\mathcal{Z}_{m}}$.

Let $(M, L)$ be a polarized Calabi-Yau manifold. We define the Teichmüller space $\mathcal{T}_{L}(M)$ to be the universal cover of $\mathcal{Z}_{m}$ with the covering map $\pi_{m}: \mathcal{T}_{L}(M) \rightarrow \mathcal{Z}_{m}$. We denote by the family $\varphi: \mathcal{U} \rightarrow \mathcal{T}_{L}(M)$ the pull-back of the family (9) by the projection $\pi_{m}$. For simplicity, we will denote $\mathcal{T}_{L}(M)$ by $\mathcal{T}$.

Proposition 2.4. The Teichmüller space $\mathcal{T}$ is a connected and simply connected smooth complex manifold and the family

$$
\varphi: \mathcal{U} \rightarrow \mathcal{T}
$$

which contains $M$ as a fiber, is local Kuranishi at each point of $\mathcal{T}$.

Proof. For the first part, because $\mathcal{Z}_{m}$ is a connected and smooth complex manifold, the universal cover of $\mathcal{Z}_{m}$ is a simply connected smooth complex manifold. For the second part, as we know that the family (9) is a versal family at each point of $\mathcal{Z}_{m}$ and that $\pi_{m}$ is locally biholomorphic, the pull-back family via $\pi_{m}$ is also versal at each point of $\mathcal{T}$. By the definition of local Kuranishi family, we get that $\mathcal{U} \rightarrow \mathcal{T}$ is local Kuranishi at each point of $\mathcal{T}$.

We remark that the family $\varphi: \mathcal{U} \rightarrow \mathcal{T}$ being local Kuranishi at each point is essentially due to the local Torelli theorem for Calabi-Yau manifolds. In fact, we know that for the family $\mathcal{U} \rightarrow \mathcal{T}$, the Kodaira-Spencer map

$$
\kappa: T_{p}^{1,0} \mathcal{T} \rightarrow H^{0,1}\left(M_{p}, T^{1,0} M_{p}\right),
$$

is an isomorphism for each $p \in \mathcal{T}$. Then by theorems in page 9 of [17], we conclude that $\mathcal{U} \rightarrow \mathcal{T}$ is versal at each $p \in \mathcal{T}$. We refer the reader to Chapter 4 in [13] for more details about deformation of complex structures and the Kodaira-Spencer map. In particular, using the unobastruction of deformation of Calabi-Yau manifolds, it is easy to see that $\operatorname{dim}_{\mathbb{C}} \mathcal{T}=\operatorname{dim}_{\mathbb{C}} H^{n-1,1}\left(M_{p}\right)=N$.

We also remark that the Teichmüller space $\mathcal{T}$ does not depend on the choice of $m$. In fact, let $m_{1}$ and $m_{2}$ be two different integers, and $\mathcal{U}_{1} \rightarrow \mathcal{T}_{1}$ and $\mathcal{U}_{2} \rightarrow \mathcal{T}_{2}$ be two versal families constructed via level $m_{1}$ and level $m_{2}$ structures respectively as above, and both of which contain $M$ as a fiber. By using the fact that $\mathcal{T}_{1}$ and $\mathcal{T}_{2}$ are simply connected and the definition of versal family, we have a biholomorphic map $f: \mathcal{T}_{1} \rightarrow \mathcal{T}_{2}$, such that the versal family $\mathcal{U}_{1} \rightarrow \mathcal{T}_{1}$ is the pull back of the versal family $\mathcal{U}_{2} \rightarrow \mathcal{T}_{2}$ by $f$. Thus these two families are biholomorphic to each other.

2.3. The period map and the Hodge metric on the Teichmüller space. For any point $p \in \mathcal{T}$, let $M_{p}$ be the fiber of family $\varphi: \mathcal{U} \rightarrow \mathcal{T}$, which is a polarized and marked Calabi-Yau manifold. Since the Teichmüller space is simply connected and we have fixed the basis of the middle homology group modulo torsions. We identify the basis of $H_{n}(M, \mathbb{Z}) /$ Tor to a lattice $\Lambda$ as in [20]. This gives us a canonical identification of the middle dimensional de Rahm cohomology of $M$ to that of the background manifold $X$, that is, $H^{n}(M) \simeq H^{n}(X)$. Therefore, we can use this 
to identify $H^{n}\left(M_{p}, \mathbb{C}\right)$ for all fibers on $\mathcal{T}$. Thus we get a canonical trivial bundle $H^{n}\left(M_{p}, \mathbb{C}\right) \times \mathcal{T}$. We have similar identifications for $H^{n}\left(M_{p}, \mathbb{Q}\right)$ and $H^{n}\left(M_{p}, \mathbb{Z}\right)$.

The period map from $\mathcal{T}$ to $D$ is defined by assigning each point $p \in \mathcal{T}$ the Hodge structure on $M_{p}$, that is

$$
\Phi: \mathcal{T} \rightarrow D, \quad p \mapsto \Phi(p)=\left\{F^{n}\left(M_{p}\right) \subset \cdots \subset F^{0}\left(M_{p}\right)\right\}
$$

We denote $F^{k}\left(M_{p}\right)$ by $F_{p}^{k}$ for simplicity.

The period map has several good properties, and we refer the reader to Chapter 10 in [24] for details. Among them, one of the most important is the following Griffiths transversality: the period map $\Phi$ is a holomorphic map and its tangent map satisfies that

$$
\Phi_{*}(v) \in \bigoplus_{k=1}^{n} \operatorname{Hom}\left(F_{p}^{k} / F_{p}^{k+1}, F_{p}^{k-1} / F_{p}^{k}\right), \quad \text { for any } \quad p \in \mathcal{T} \text { and } v \in T_{p}^{1,0} \mathcal{T}
$$

with $F^{n+1}=0$, or equivalently, $\Phi_{*}(v) \in \bigoplus_{k=0}^{n} \operatorname{Hom}\left(F_{p}^{k}, F_{p}^{k-1}\right)$.

In [5], Griffiths and Schmid studied the so-called Hodge metric on the period domain $D$. We denote it by $h$. In particular, this Hodge metric is a complete homogeneous metric. Let us denote the period map on the moduli space by $\Phi_{\mathcal{Z}_{m}}: \mathcal{Z}_{m} \rightarrow D / \Gamma$, where $\Gamma$ denotes the global monodromy group which acts properly and discontinuously on the period domain $D$. Since $\pi_{m}: \mathcal{T} \rightarrow \mathcal{Z}_{m}$ is the universal covering map, the period map $\Phi: \mathcal{T} \rightarrow D$ is the lifting of $\Phi_{\mathcal{Z}_{m}} \circ \pi_{m}$. By local Torelli theorem for Calabi-Yau manifolds, we know that $\Phi_{\mathcal{Z}_{m}}$ is locally injective and $\Phi$ is also locally injective. Thus it follows from [5] that the pull-backs of $h$ by $\Phi_{\mathcal{Z}_{m}}$ and $\Phi$ on $\mathcal{Z}_{m}$ and $\mathcal{T}$ respectively are both well-defined Kähler metrics. By abuse of notation, we still call these pull-back metrics the Hodge metrics, and they are both denoted by $h$.

3. Holomorphic affine structure on the Teichmüller space. In Section 3.1, we review some properties of the period domain from Lie group and Lie algebra point of view. In Section 3.2, we fix a base point $p \in \mathcal{T}$ and introduce the unipotent space $N_{+} \subseteq \check{D}$, which is biholomorphic to $\mathbb{C}^{d}$. Then we show that the image $\Phi(\mathcal{T})$ is bounded in $N_{+} \cap D$ with respect to the Euclidean metric on $N_{+}$. In Section 3.3, using the property that $\Phi(\mathcal{T}) \subseteq N_{+}$, we define a holomorphic map $\tau: \mathcal{T} \rightarrow \mathbb{C}^{N}$. Then we use local Torelli theorem to show that $\tau$ defines a local coordinate chart around each point in $\mathcal{T}$, and this shows that $\tau: \mathcal{T} \rightarrow \mathbb{C}^{N}$ defines a holomorphic affine structure on $\mathcal{T}$.

3.1. Preliminary. Let us briefly recall some properties of the period domain from Lie group and Lie algebra point of view. All of the results in this section is wellknown to the experts in the subject. The purpose to give details is to fix notations. One may either skip this section or refer to [5] and [16] for most of the details.

The orthogonal group of the bilinear form $Q$ in the definition of Hodge structure is a linear algebraic group, defined over $\mathbb{Q}$. Let us simply denote $H_{\mathbb{C}}=H^{n}(M, \mathbb{C})$ and $H_{\mathbb{R}}=H^{n}(M, \mathbb{R})$. The group of the $\mathbb{C}$-rational points is

$$
G_{\mathbb{C}}=\left\{g \in G L\left(H_{\mathbb{C}}\right) \mid Q(g u, g v)=Q(u, v) \text { for all } u, v \in H_{\mathbb{C}}\right\},
$$

which acts on $\check{D}$ transitively. The group of real points in $G_{\mathbb{C}}$ is

$$
G_{\mathbb{R}}=\left\{g \in G L\left(H_{\mathbb{R}}\right) \mid Q(g u, g v)=Q(u, v) \text { for all } u, v \in H_{\mathbb{R}}\right\},
$$


which acts transitively on $D$ as well.

Consider the period map $\Phi: \mathcal{T} \rightarrow D$. Fix a point $p \in \mathcal{T}$ with the image $o:=\Phi(p)=\left\{F_{p}^{n} \subset \cdots \subset F_{p}^{0}\right\} \in D$. The points $p \in \mathcal{T}$ and $o \in D$ may be referred as the base points or the reference points. A linear transformation $g \in G_{\mathbb{C}}$ preserves the base point if and only if $g F_{p}^{k}=F_{p}^{k}$ for each $k$. Thus it gives the identification

$$
\check{D} \simeq G_{\mathbb{C}} / B \quad \text { with } \quad B=\left\{g \in G_{\mathbb{C}} \mid g F_{p}^{k}=F_{p}^{k}, \text { for any } k\right\} .
$$

Similarly, one obtains an analogous identification

$$
D \simeq G_{\mathbb{R}} / V \hookrightarrow \check{D} \text { with } \quad V=G_{\mathbb{R}} \cap B,
$$

where the embedding corresponds to the inclusion $G_{\mathbb{R}} / V=G_{\mathbb{R}} / G_{\mathbb{R}} \cap B \subseteq G_{\mathbb{C}} / B$. The Lie algebra $\mathfrak{g}$ of the complex Lie group $G_{\mathbb{C}}$ can be described as

$$
\mathfrak{g}=\left\{X \in \operatorname{End}\left(H_{\mathbb{C}}\right) \mid Q(X u, v)+Q(u, X v)=0, \text { for all } u, v \in H_{\mathbb{C}}\right\}
$$

It is a simple complex Lie algebra, which contains $\mathfrak{g}_{0}=\left\{X \in \mathfrak{g} \mid X H_{\mathbb{R}} \subseteq H_{\mathbb{R}}\right\}$ as a real form, i.e. $\mathfrak{g}=\mathfrak{g}_{0} \oplus i \mathfrak{g}_{0}$. With the inclusion $G_{\mathbb{R}} \subseteq G_{\mathbb{C}}, \mathfrak{g}_{0}$ becomes Lie algebra of $G_{\mathbb{R}}$. One observes that the reference Hodge structure $\left\{H_{p}^{k, n-k}\right\}_{k=0}^{n}$ of $H^{n}(M, \mathbb{C})$ induces a Hodge structure of weight zero on $\operatorname{End}\left(H^{n}(M, \mathbb{C})\right)$, namely,

$$
\mathfrak{g}=\bigoplus_{k \in \mathbb{Z}} \mathfrak{g}^{k,-k} \quad \text { with } \quad \mathfrak{g}^{k,-k}=\left\{X \in \mathfrak{g} \mid X H_{p}^{r, n-r} \subseteq H_{p}^{r+k, n-r-k}\right\}
$$

Since the Lie algebra $\mathfrak{b}$ of $B$ consists of those $X \in \mathfrak{g}$ that preserves the reference Hodge filtration $\left\{F_{p}^{n} \subset \cdots \subset F_{p}^{0}\right\}$, one thus has

$$
\mathfrak{b}=\bigoplus_{k \geq 0} \mathfrak{g}^{k,-k}
$$

The Lie algebra $\mathfrak{v}_{0}$ of $V$ is $\mathfrak{v}_{0}=\mathfrak{g}_{0} \cap \mathfrak{b}=\mathfrak{g}_{0} \cap \mathfrak{b} \cap \overline{\mathfrak{b}}=\mathfrak{g}_{0} \cap \mathfrak{g}^{0,0}$. With the above isomorphisms, the holomorphic tangent space of $\check{D}$ at the base point is naturally isomorphic to $\mathfrak{g} / \mathfrak{b}$.

Let us consider the nilpotent Lie subalgebra $\mathfrak{n}_{+}:=\oplus_{k \geq 1} \mathfrak{g}^{-k, k}$. Then one gets the holomorphic isomorphism $\mathfrak{g} / \mathfrak{b} \cong \mathfrak{n}_{+}$. We take the unipotent group $N_{+}=\exp \left(\mathfrak{n}_{+}\right)$.

$\operatorname{As} \operatorname{Ad}(g)\left(\mathfrak{g}^{k,-k}\right)$ is in $\bigoplus_{i \geq k} \mathfrak{g}^{i,-i}$ for each $g \in B$, the sub-Lie algebra $\mathfrak{b} \oplus \mathfrak{g}^{-1,1} / \mathfrak{b} \subseteq$ $\mathfrak{g} / \mathfrak{b}$ defines an $\operatorname{Ad}(B)$-invariant subspace. By left translation via $G_{\mathbb{C}}, \mathfrak{b} \oplus \mathfrak{g}^{-1,1} / \mathfrak{b}$ gives

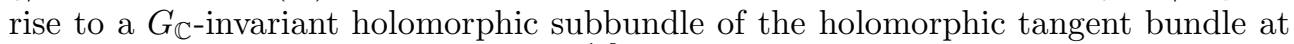
the base point. It will be denoted by $T_{o, h}^{1,0} \check{D}$, and will be referred to as the holomorphic horizontal tangent bundle at the base point. One can check that this construction does not depend on the choice of the base point. The horizontal tangent subbundle at the base point $o$, restricted to $D$, determines a subbundle $T_{o, h}^{1,0} D$ of the holomorphic

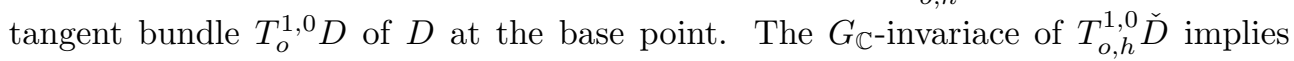
the $G_{\mathbb{R}^{-}}$invariance of $T_{o, h}^{1,0} D$. As another interpretation of this holomorphic horizontal bundle at the base point, one has

$$
T_{o, h}^{1,0} \check{D} \simeq T_{o}^{1,0} \check{D} \cap \bigoplus_{k=1}^{n} \operatorname{Hom}\left(F_{p}^{k} / F_{p}^{k+1}, F_{p}^{k-1} / F_{p}^{k}\right) .
$$

In [16], Schmid call a holomorphic mapping $\Psi: M \rightarrow \check{D}$ of a complex manifold $M$ into $D$ horizontal if at each point of $M$, the induced map between the holomorphic 
tangent spaces takes values in the appropriate fibre $T^{1,0} \check{D}$. It is easy to see that the period map $\Phi: \mathcal{T} \rightarrow D$ is horizontal since $\Phi_{*}\left(T_{p}^{1,0} \mathcal{T}\right) \subseteq T_{o, h}^{1,0} D$ for any $p \in \mathcal{T}$. Since $D$ is an open set in $\check{D}$, we have the following relation:

$$
T_{o, h}^{1,0} D=T_{o, h}^{1,0} \check{D} \cong \mathfrak{b} \oplus \mathfrak{g}^{-1,1} / \mathfrak{b} \hookrightarrow \mathfrak{g} / \mathfrak{b} \cong \mathfrak{n}_{+} .
$$

REMARK 3.1. With a fixed base point, we can identify $N_{+}$with its unipotent orbit in $\check{D}$ by identifying an element $c \in N_{+}$with $[c]=c B$ in $\check{D}$; that is, $N_{+}=$ $N_{+}$( base point $) \cong N_{+} B / B \subseteq \check{D}$. In particular, when the base point $o$ is in $D$, we have $N_{+} \cap D \subseteq D$.

Let us introduce the notion of an adapted basis for the given Hodge decomposition or the Hodge filtration. For any $p \in \mathcal{T}$ and $f^{k}=\operatorname{dim} F_{p}^{k}$ for any $0 \leq k \leq n$, we call a basis

$$
\xi=\left\{\xi_{0}, \xi_{1}, \cdots, \xi_{N}, \cdots, \xi_{f^{k+1}}, \cdots, \xi_{f^{k}-1}, \cdots, \xi_{f^{2}}, \cdots, \xi_{f^{1}-1}, \xi_{f^{0}-1}\right\}
$$

of $H^{n}\left(M_{p}, \mathbb{C}\right)$ an adapted basis for the given Hodge decomposition

$$
H^{n}\left(M_{p}, \mathbb{C}\right)=H_{p}^{n, 0} \oplus H_{p}^{n-1,1} \oplus \cdots \oplus H_{p}^{1, n-1} \oplus H_{p}^{0, n},
$$

if it satisfies $H_{p}^{k, n-k}=\operatorname{Span}_{\mathbb{C}}\left\{\xi_{f^{k+1}}, \cdots, \xi_{f^{k}-1}\right\}$ with $\operatorname{dim} H_{p}^{k, n-k}=f^{k}-f^{k+1}$. We call a basis

$$
\zeta=\left\{\zeta_{0}, \zeta_{1}, \cdots, \zeta_{N}, \cdots, \zeta_{f^{k+1}}, \cdots, \zeta_{f^{k}-1}, \cdots, \zeta_{f^{2}}, \cdots, \zeta_{f^{1}-0}, \zeta_{f^{0}-1}\right\}
$$

of $H^{n}\left(M_{p}, \mathbb{C}\right)$ an adapted basis for the given filtration

$$
F^{n} \subseteq F^{n-1} \subseteq \cdots \subseteq F^{0}
$$

if it satisfies $F^{k}=\operatorname{Span}_{\mathbb{C}}\left\{\zeta_{0}, \cdots, \zeta_{f^{k}-1}\right\}$ with $\operatorname{dim}_{\mathbb{C}} F^{k}=f^{k}$. Moreover, unless otherwise pointed out, the matrices in this paper are $m \times m$ matrices, where $m=f^{0}$. The blocks of the $m \times m$ matrix $T$ is set as follows: for each $0 \leq \alpha, \beta \leq n$, the $(\alpha, \beta)$-th block $T^{\alpha, \beta}$ is

$$
T^{\alpha, \beta}=\left[T_{i j}(\tau)\right]_{f^{-\alpha+n+1} \leq i \leq f^{-\alpha+n}-1, f^{-\beta+n+1} \leq j \leq f^{-\beta+n}-1},
$$

where $T_{i j}$ is the entries of the matrix $T$, and $f^{n+1}$ is defined to be zero. In particular, $T=\left[T^{\alpha, \beta}\right]$ is called a block lower triangular matrix if $T^{\alpha, \beta}=0$ whenever $\alpha<\beta$.

REMARK 3.2. We remark that by fixing a base point, we can identify the above quotient Lie groups or Lie algebras with their orbits in the corresponding quotient Lie algebras or Lie groups. For example, $\mathfrak{n}_{+} \cong \mathfrak{g} / \mathfrak{b}, \mathfrak{g}^{-1,1} \cong \mathfrak{b} \oplus \mathfrak{g}^{-1,1} / \mathfrak{b}$, and $N_{+} \cong$ $N_{+} B / B \subseteq \check{D}$. We can also identify a point $\Phi(p)=\left\{F_{p}^{n} \subseteq F_{p}^{n-1} \subseteq \cdots \subseteq F_{p}^{0}\right\} \in$ $D$ with its Hodge decomposition $\bigoplus_{k=0}^{n} H_{p}^{k, n-k}$, and thus with any fixed adapted basis of the corresponding Hodge decomposition for the base point, we have matrix representations of elements in the above Lie groups and Lie algebras. For example, elements in $N_{+}$can be realized as nonsingular block lower triangular matrices with identity blocks in the diagonal; elements in $B$ can be realized as nonsingular block upper triangular matrices. 
We shall review and collect some facts about the structure of simple Lie algebra $\mathfrak{g}$ in our case. Again one may refer to [5] and [16] for more details. Let $\theta: \mathfrak{g} \rightarrow \mathfrak{g}$ be the Weil operator, which is defined by

$$
\theta(X)=(-1)^{p} X \quad \text { for } X \in \mathfrak{g}^{p,-p} .
$$

Then $\theta$ is an involutive automorphism of $\mathfrak{g}$, and is defined over $\mathbb{R}$. The $(+1)$ and $(-1)$ eigenspaces of $\theta$ will be denoted by $\mathfrak{k}$ and $\mathfrak{p}$ respectively. Moreover, set

$$
\mathfrak{k}_{0}=\mathfrak{k} \cap \mathfrak{g}_{0}, \quad \mathfrak{p}_{0}=\mathfrak{p} \cap \mathfrak{g}_{0} .
$$

The fact that $\theta$ is an involutive automorphism implies

$$
\mathfrak{g}=\mathfrak{k} \oplus \mathfrak{p}, \quad \mathfrak{g}_{0}=\mathfrak{k}_{0} \oplus \mathfrak{p}_{0}, \quad[\mathfrak{k}, \mathfrak{k}] \subseteq \mathfrak{k}, \quad[\mathfrak{p}, \mathfrak{p}] \subseteq \mathfrak{p}, \quad[\mathfrak{k}, \mathfrak{p}] \subseteq \mathfrak{p} .
$$

Let us consider $\mathfrak{g}_{c}=\mathfrak{k}_{0} \oplus \sqrt{-1} \mathfrak{p}_{0}$. Then $\mathfrak{g}_{c}$ is a real form for $\mathfrak{g}$. Recall that the killing form $B(\cdot, \cdot)$ on $\mathfrak{g}$ is defined by

$$
B(X, Y)=\operatorname{Trace}(\operatorname{ad}(X) \circ \operatorname{ad}(Y)) \quad \text { for } X, Y \in \mathfrak{g} .
$$

A semisimple Lie algebra is compact if and only if the Killing form is negative definite. Thus it is not hard to check that $\mathfrak{g}_{c}$ is actually a compact real form of $\mathfrak{g}$, while $\mathfrak{g}_{0}$ is a non-compact real form. Recall that $G_{\mathbb{R}} \subseteq G_{\mathbb{C}}$ is the subgroup which correpsonds to the subalgebra $\mathfrak{g}_{0} \subseteq \mathfrak{g}$. Let us denote the connected subgroup $G_{c} \subseteq G_{\mathbb{C}}$ which corresponds to the subalgebra $\mathfrak{g}_{c} \subseteq \mathfrak{g}$. Let us denote the complex conjugation of $\mathfrak{g}$ with respect to the compact real form by $\tau_{c}$, and the complex conjugation of $\mathfrak{g}$ with respect to the compact real form by $\tau_{0}$.

The intersection $K=G_{c} \cap G_{\mathbb{R}}$ is then a compact subgroup of $\mathbb{G}_{\mathbb{R}}$, whose Lie algebra is $\mathfrak{k}_{0}=\mathfrak{g}_{\mathbb{R}} \cap \mathfrak{g}_{c}$. With the above notations, Schmid showed in [16] that $K$ is a maximal compact subgroup of $G_{\mathbb{R}}$, and it meets every connected component of $G_{\mathbb{R}}$. Moreover, $V=G_{\mathbb{R}} \cap B \subseteq K$. As remarked in $\S 1$ in [5] of Griffiths and Schmid, one gets that $\mathfrak{v}$ must have the same rank of $\mathfrak{g}$ as $\mathfrak{v}$ is the intersection of the two parabolic subalgebras $\mathfrak{b}$ and $\tau_{c}(\mathfrak{b})$. Moreover, $\mathfrak{g}_{0}$ and $\mathfrak{v}_{0}$ are also of equal rank, since they are real forms of $\mathfrak{g}$ and $\mathfrak{v}$ respectively. Therefore, we have the following proposition.

Proposition 3.3. There exists a Cartan subalgebra $\mathfrak{h}_{0}$ of $\mathfrak{g}_{0}$ such that $\mathfrak{h}_{0} \subseteq \mathfrak{v}_{0} \subseteq$ $\mathfrak{k}_{0}$ and $\mathfrak{h}_{0}$ is also a Cartan subalgebra of $\mathfrak{k}_{0}$.

Proposition 3.3 implies that the simple Lie algebra $\mathfrak{g}_{0}$ in our case is a simple Lie algebra of first category as defined in $\S 4$ in [18]. In the upcoming part, we will briefly derive the result of a simple Lie algebra of first category in Lemma 3 in [19]. One may also refer to [25] Lemma 2.2.12 at pp. 141-142 for the same result.

Let us still use the above notations of the Lie algebras we consider. By Proposition 4, we can take $\mathfrak{h}_{0}$ to be a Cartan subalgebra of $\mathfrak{g}$ such that $\mathfrak{h}_{0} \subseteq \mathfrak{v}_{0} \subseteq \mathfrak{k}_{0}$ and $\mathfrak{h}_{0}$ is also a Cartan subalgebra of $\mathfrak{k}_{0}$. Let us denote $\mathfrak{h}$ to be the complexification of $\mathfrak{h}_{0}$. Then $\mathfrak{h}$ is a Cartan subalgebra of $\mathfrak{g}$ such that $\mathfrak{h} \subseteq \mathfrak{v} \subseteq \mathfrak{k}$.

Write $\mathfrak{h}_{0}^{*}=\operatorname{Hom}\left(\mathfrak{h}_{0}, \mathbb{R}\right)$ and $\mathfrak{h}_{\mathbb{R}}^{*}=\sqrt{-1} \mathfrak{h}_{0}^{*}$. Then $\mathfrak{h}_{\mathbb{R}}^{*}$ can be identified with $\mathfrak{h}_{\mathbb{R}}:=$ $\sqrt{-1} \mathfrak{h}_{0}$ by duality using the restriction of the Killing form $B$ of $\mathfrak{g}$ to $\mathfrak{h}_{\mathbb{R}}$. Let $\rho \in \mathfrak{h}_{\mathbb{R}}^{*} \simeq$ $\mathfrak{h}_{\mathbb{R}}$, one can define the following subspace of $\mathfrak{g}$

$$
\mathfrak{g}^{\rho}=\{x \in \mathfrak{g} \mid[h, x]=\rho(h) x \quad \text { for all } h \in \mathfrak{h}\} .
$$

An element $\varphi \in \mathfrak{h}_{\mathbb{R}}^{*} \simeq \mathfrak{h}_{\mathbb{R}}$ is called a root of $\mathfrak{g}$ with respect to $\mathfrak{h}$ if $\mathfrak{g}^{\varphi} \neq\{0\}$. 
Let $\Delta \subseteq \mathfrak{h}_{\mathbb{R}}^{*} \simeq \mathfrak{h}_{\mathbb{R}}$ denote the space of nonzero $\mathfrak{h}$-roots. Then each root space

$$
\mathfrak{g}^{\varphi}=\{x \in \mathfrak{g} \mid[h, x]=\varphi(h) x \text { for all } h \in \mathfrak{h}\}
$$

belongs to some $\varphi \in \Delta$ is one-dimensional over $\mathbb{C}$, generated by a root vector $e_{\varphi}$.

Since the involution $\theta$ is a Lie-algebra automorphism fixing $\mathfrak{k}$, we have $\left[h, \theta\left(e_{\varphi}\right)\right]=$ $\varphi(h) \theta\left(e_{\varphi}\right)$ for any $h \in \mathfrak{h}$ and $\varphi \in \Delta$. Thus $\theta\left(e_{\varphi}\right)$ is also a root vector belonging to the root $\varphi$, so $e_{\varphi}$ must be an eigenvector of $\theta$. It follows that there is a decomposition of the roots $\Delta$ into $\Delta_{\mathfrak{k}} \cup \Delta_{\mathfrak{p}}$ of compact roots and non-compact roots with root spaces $\mathbb{C} e_{\varphi} \subseteq \mathfrak{k}$ and $\mathfrak{p}$ respectively. The adjoint representation of $\mathfrak{h}$ on $\mathfrak{g}$ determins a decomposition

$$
\mathfrak{g}=\mathfrak{h} \oplus \sum_{\varphi \in \Delta} \mathfrak{g}^{\varphi} .
$$

There also exists a Weyl base $\left\{h_{i}, 1 \leq i \leq l\right.$; $e_{\varphi}$, for any $\left.\varphi \in \Delta\right\}$ with $l=\operatorname{rank}(\mathfrak{g})$ such that $\operatorname{Span}_{\mathbb{C}}\left\{h_{1}, \cdots, h_{l}\right\}=\mathfrak{h}, \operatorname{Span}_{\mathbb{C}}\left\{e_{\varphi}\right\}=g^{\varphi}$ for each $\varphi \in \Delta$, and

$$
\begin{aligned}
& \tau_{c}\left(h_{i}\right)=\tau_{0}\left(h_{i}\right)=-h_{i}, \quad \text { for any } 1 \leq i \leq l ; \\
\tau_{c}\left(e_{\varphi}\right)= & \tau_{0}\left(e_{\varphi}\right)=-e_{-\varphi} \quad \text { for any } \varphi \in \Delta_{\mathfrak{k}} ; \quad \tau_{0}\left(e_{\varphi}\right)=-\tau_{c}\left(e_{\varphi}\right)=e_{\varphi} \quad \text { for any } \varphi \in \Delta_{\mathfrak{p}} .
\end{aligned}
$$

With respect to this Weyl base, we have

$$
\begin{aligned}
\mathfrak{k}_{0} & =\mathfrak{h}_{0}+\sum_{\varphi \in \Delta_{\mathfrak{k}}} \mathbb{R}\left(e_{\varphi}-e_{-\varphi}\right)+\sum_{\varphi \in \Delta_{\mathfrak{k}}} \mathbb{R} \sqrt{-1}\left(e_{\varphi}+e_{-\varphi}\right) ; \\
\mathfrak{p}_{0} & =\sum_{\varphi \in \Delta_{\mathfrak{p}}} \mathbb{R}\left(e_{\varphi}+e_{-\varphi}\right)+\sum_{\varphi \in \Delta_{\mathfrak{p}}} \mathbb{R} \sqrt{-1}\left(e_{\varphi}-e_{-\varphi}\right) .
\end{aligned}
$$

Let us now introduce a lexicographic order (cf. pp.41 in [25] or pp.416 in [18]) in the real vector space $\mathfrak{h}_{\mathbb{R}}$ as follows: we fix an ordered basis $e_{1}, \cdots, e_{l}$ for $\mathfrak{h}_{\mathbb{R}}$. Then for any $h=\sum_{i=1}^{l} \lambda_{i} e_{i} \in \mathfrak{h}_{\mathbb{R}}$, we call $h>0$ if the first nonzero coefficient is positive, that is, if $\lambda_{1}=\cdots=\lambda_{k}=0, \lambda_{k+1}>0$ for some $1 \leq k<l$. For any $h, h^{\prime} \in \mathfrak{h}_{\mathbb{R}}$, we say $h>h^{\prime}$ if $h-h^{\prime}>0, h<h^{\prime}$ if $h-h^{\prime}<0$ and $h=h^{\prime}$ if $h-h^{\prime}=0$. Now let us first choose a maximal linearly independent subset $S=\left\{s_{1}, \cdots, s_{k}\right\}$ of $\Delta_{\mathfrak{k}}$, and then choose a linearly independent subset $E=\left\{e_{1}, \cdots, e_{l-k}\right\}$ of $\Delta_{\mathfrak{p}}$ such that $E \cup S$ is a basis for $\mathfrak{h}_{\mathbb{R}}^{*}$, where $l$ is the real dimension of $\mathfrak{h}_{\mathbb{R}}^{*}$. Now we order this basis $E \cup S$ as $\left\{e_{1}, \cdots, e_{l-k}, s_{1}, \cdots, s_{k}\right\}$, namely, we put the noncompact roots in front of the compact ones. Then we define the above lexicographic order in $\mathfrak{h}_{\mathbb{R}}^{*} \simeq \mathfrak{h}_{\mathbb{R}}$. Then we define $\Delta^{ \pm}, \Delta_{\mathfrak{p}}^{ \pm}$, and $\Delta_{\mathfrak{k}}^{ \pm}$.

DeFinition 3.4. Two different roots $\varphi, \psi \in \Delta$ are said to be strongly orthogonal if and only if $\varphi \pm \psi \notin \Delta \cup\{0\}$, which is denoted by $\varphi \Perp \psi$.

For the real simple Lie algebra $\mathfrak{g}_{0}=\mathfrak{k}_{0} \oplus \mathfrak{p}_{0}$ which has a Cartan subalgebra $\mathfrak{h}_{0}$ in $\mathfrak{k}_{0}$, the maximal abelian subspace of $\mathfrak{p}_{0}$ can be described as in the following lemma, which is a slight extension of a lemma of Harish-Chandra in [6]. One may refer to Lemma 3 in [19] or Lemma 2.2.12 at pp.141-142 in [25] for more details. For reader's convenience we give the detailed proof.

LEMMA 3.5. There exists a set of strongly orthogonal noncompact positive roots $\Lambda=\left\{\varphi_{1}, \cdots, \varphi_{r}\right\} \subseteq \Delta_{\mathfrak{p}}^{+}$such that

$$
\mathfrak{a}_{0}=\sum_{i=1}^{r} \mathbb{R}\left(e_{\varphi_{i}}+e_{-\varphi_{i}}\right)
$$


is a maximal abelian subspace in $\mathfrak{p}_{0}$.

This lemma is a slight extension of a lemma of Harish-Chandra in [6]. One may refer to Lemma 3 in [19] or Lemma 2.2.12 at pp.141-142 in [25] for more details. One may also find the detailed proof in [1].

3.2. Boundedness of the period map. Now let us fix the base point $p \in \mathcal{T}$ with $\Phi(p) \in D$. Then according to the above remark, $N_{+}$can be viewed as a subset in $\check{D}$ by identifying it with its orbit in $\check{D}$ with base point $\Phi(p)$. Let us also fix an adapted basis $\left(\eta_{0}, \cdots, \eta_{m-1}\right)$ for the Hodge decomposition of the base point $\Phi(p) \in D$. Then we can identify elements in $N_{+}$with nonsingular block lower triangular matrices whose diagonal blocks are all identity submatrix. We define

$$
\check{\mathcal{T}}=\Phi^{-1}\left(N_{+}\right)
$$

At the base point $\Phi(p)=o \in N_{+} \cap D$, the tangent space $T_{o}^{1,0} N_{+}=T_{o}^{1,0} D \simeq \mathfrak{n}_{+} \simeq N_{+}$, then the Hodge metric on $T_{o}^{1,0} D$ induces an Euclidean metric on $N_{+}$. In the proof of the following lemma, we require all the root vectors to be unit vectors with respect to this Euclidean metric.

Because the period map is a horizontal map, and the geometry of horizontal slices of the period domain $D$ is similar to Hermitian symmetric space as discussed in detail in [5], the proof the following theorem is basically an analogue of the proof of the Harish-Chandra embedding theorem for Hermitian symmetric spaces, see for example [10].

THEOREM 3.6. The restriction of the period map $\Phi: \check{\mathcal{T}} \rightarrow N_{+}$is bounded in $N_{+}$ with respect to the Euclidean metric on $N_{+}$.

The decomposition of $\mathfrak{p}$ in Lemma 3.5 along with the property that the period map is horizontal allows us to show that $\Phi(\check{\mathcal{T}})$ sits in a polydisc, a product of discs of radius 1 up to normalization, in $N_{+}$. This argument is a slight extension of HarishChandra's proof of his famous embedding theorem of the Hermitian symmetric domains as bounded domains in complex Euclidean spaces. One may refer to Lemma 7 and Lemma 8 at pp. 582-583 in [6], Proposition 7.4 at pp. 385 and Ch VIII $\S 7$ at pp. 382-396 in [7], Proposition 1 at pp. 91 and Proof of Theorem 1 at pp. 95-97 in [10], and Lemma 2.2.12 at pp. 141-142 and $\$ 5.4$ in [25] for more details.

According to the definition of $\check{\mathcal{T}}$, it is not hard to conclude the following lemma, one may refer to [1] for the complete proof.

LEMma 3.7. The subset $\check{\mathcal{T}}$ is an open dense submanifold in $\mathcal{T}$, and $\mathcal{T} \backslash \check{\mathcal{T}}$ is an analytic subvariety of $\mathcal{T}$ with $\operatorname{codim}_{\mathbb{C}}(\mathcal{T} \backslash \check{\mathcal{T}}) \geq 1$.

Corollary 3.8. The image of $\Phi: \mathcal{T} \rightarrow D$ lies in $N_{+} \cap D$ and is bounded with respect to the Euclidean metric on $N_{+}$.

Proof. According to Lemma 3.7, $\mathcal{T} \backslash \check{\mathcal{T}}$ is an analytic subvariety of $\mathcal{T}$ and the complex codimension of $\mathcal{T} \backslash \check{\mathcal{T}}$ is at least one; by Theorem3.6, the holomorphic map $\Phi: \check{\mathcal{T}} \rightarrow N_{+} \cap D$ is bounded in $N_{+}$with respect to the Euclidean metric. Thus by the Riemann extension theorem, there exists a holomorphic map $\Phi^{\prime}: \mathcal{T} \rightarrow N_{+} \cap D$ such that $\left.\Phi^{\prime}\right|_{\check{\mathcal{T}}}=\left.\Phi\right|_{\check{\mathcal{T}}}$. Since as holomorphic maps, $\Phi^{\prime}$ and $\Phi$ agree on the open subset $\check{\mathcal{T}}$, they must be the same on the entire $\mathcal{T}$. Therefore, the image of $\Phi$ is in $N_{+} \cap D$, and the image is bounded with respect to the Euclidean metric on $N_{+}$. As a consequence, we also get $\mathcal{T}=\check{\mathcal{T}}=\Phi^{-1}\left(N_{+}\right)$. 
3.3. Holomorphic affine structure on the Teichmüller space. We first review the definition of complex affine manifolds. One may refer to page 215 of [14] for more details.

Definition 3.9. Let $M$ be a complex manifold of complex dimension $n$. If there is a coordinate cover $\left\{\left(U_{i}, \varphi_{i}\right) ; i \in I\right\}$ of $M$ such that $\varphi_{i k}=\varphi_{i} \circ \varphi_{k}^{-1}$ is a holomorphic affine transformation on $\mathbb{C}^{n}$ whenever $U_{i} \cap U_{k}$ is not empty, then $\left\{\left(U_{i}, \varphi_{i}\right) ; i \in I\right\}$ is called a complex affine coordinate cover on $M$ and it defines a holomorphic affine structure on $M$.

Let us still fix an adapted basis $\left(\eta_{0}, \cdots, \eta_{m-1}\right)$ for the Hodge decomposition of the base point $\Phi(p) \in D$. Recall that we can identify elements in $N_{+}$with nonsingular block lower triangular matrices whose diagonal blocks are all identity submatrix, and element in $B$ with nonsingular block upper triangular matrices. Therefore $N_{+} \cap$ $B=I d$. By Corollary 3.8, we know that $\mathcal{T}=\Phi^{-1}\left(N_{+}\right)$. Thus we get that each $\Phi(q)$ can be uniquely determined by a matrix, which we will still denote by $\Phi(q)=$ $\left[\Phi_{i j}(q)\right]_{0 \leq i, j \leq m-1}$ of the form of nonsingular block lower triangular matrices whose diagonal blocks are all identity submatrix. Thus we can define a holomorphic map

$$
\begin{array}{ll}
\tau: \mathcal{T} \rightarrow \mathbb{C}^{N} \cong H_{p}^{n-1,1}, \quad q \mapsto(1,0) \text {-block of the matrix } \Phi(q) \in N_{+}, \\
\text {that is } \quad \tau(q)=\left(\tau_{1}(q), \tau_{2}(q), \cdots, \tau_{N}(q)\right)=\left(\Phi_{10}(q), \Phi_{20}(q), \cdots, \Phi_{N 0}(q)\right)
\end{array}
$$

REMARK 3.10. If we define the following projection map with respect to the base point and the its pre-fixed adapted basis to the Hodge decomposition,

$$
P: N_{+} \cap D \rightarrow H_{p}^{n-1,1} \cong \mathbb{C}^{N}, \quad F \mapsto\left(\eta_{1}, \cdots, \eta_{N}\right) F^{(1,0)}=F_{10} \eta_{1}+\cdots+F_{N 0} \eta_{N},
$$

where $F^{(1,0)}$ is the $(1,0)$-block of the unipotent matrix $F$, according to our convention in (13), then $\tau=P \circ \Phi: \mathcal{T} \rightarrow \mathbb{C}^{N}$.

Proposition 3.11. The holomorphic map $\tau=\left(\tau_{1}, \cdots, \tau_{N}\right): \mathcal{T} \rightarrow \mathbb{C}^{N}$ defines a coordinate chart around each point $q \in \mathcal{T}$.

To prove that $\tau$ defines a coordinate map around each point $q \in \mathcal{T}$, it suffices to show that the tangent map $\tau_{*}: T_{q}^{1,0} \mathcal{T} \rightarrow T_{\tau(q)} \mathbb{C}^{N}$ is an isomorphism. This essentially follows from the local Torelli theorem for Calabi-Yau manifolds. Indeed, we have the generator $\Omega(p)=\eta_{0} \in H^{0}\left(M_{p}, \Omega^{n}\left(M_{p}\right)\right)$, the generators $\left\{\eta_{1}, \cdots, \eta_{N}\right\} \subseteq H^{1}\left(M_{p}, \Omega^{n-1}\left(M_{p}\right)\right)$, and the generators $\left\{\eta_{N+1}, \cdots, \eta_{m-1}\right\} \subseteq$ $\bigoplus_{k \geq 2} H^{k}\left(M_{p}, \Omega^{n-k}\left(M_{p}\right)\right.$.

On one hand, the 0 -th column of the matrix $\Phi(q) \in N_{+}$for each $q \in \mathcal{T}$ gives us the following data:

$$
\begin{aligned}
\Omega & : \mathcal{T} \rightarrow F^{n} \\
\Omega(q) & =\left(\eta_{0}, \cdots, \eta_{m-1}\right)\left(\Phi_{00}(q), \Phi_{10}(q), \Phi_{20}(q), \cdots, \cdots\right)^{T} \\
& =\eta_{0}+\tau_{1}(q) \eta_{1}+\tau_{2}(q) \eta_{2}+\cdots \tau_{N}(q) \eta_{N}+g_{0}(q) \in F_{q}^{n} \simeq H^{0}\left(M_{q}, \Omega^{n}\left(M_{q}\right)\right),
\end{aligned}
$$

where $g_{0}(q) \in \bigoplus_{k \geq 2} H^{k}\left(M_{p}, \Omega^{n-k}\left(M_{p}\right)\right)$.

The 1 -st to $N$-th columns of $\Phi(q) \in N_{+}$give us the following data:

$$
\theta_{1}(q)=\eta_{1}+g_{1}(q), \quad \cdots \cdots, \quad \theta_{N}(q)=\eta_{N}+g_{N}(q) \in F_{q}^{n-1},
$$


where $g_{k}(q) \in \bigoplus_{k>2} H^{k}\left(M_{p}, \Omega^{n-k}\left(M_{p}\right)\right)$, such that $\left\{\Omega(q), \theta_{1}(q), \cdots, \theta_{N}(q)\right\}$ forms a basis for $F_{q}^{n-1}$ for each $q \in \mathcal{T}$.

On the other hand, by local Torelli theorem, we know that for any holomorphic coordinate $\left\{\sigma_{1}, \cdots, \sigma_{N}\right\}$ around $q,\left\{\Omega(q), \frac{\partial \Omega(q)}{\partial \sigma_{1}}, \cdots, \frac{\partial \Omega(q)}{\partial \sigma_{N}}\right\}$ forms a basis of $F_{q}^{n-1}$.

Now by using the fact that $\left\{\Omega(q), \theta_{1}(q), \cdots, \theta_{N}(q)\right\}$ and $\left\{\Omega(q), \frac{\partial \Omega(q)}{\partial \sigma_{1}}, \cdots, \frac{\partial \Omega(q)}{\partial \sigma_{N}}\right\}$ are both bases for $F_{q}^{n-1}$, and that $\theta_{k}$ doesn't involve components in $H^{0}\left(M_{p}, \Omega^{n}\left(M_{p}\right)\right)$, we may assume that

$$
\frac{\partial \Omega(q)}{\partial \sigma_{k}}=\theta_{k}=\eta_{k}+g_{k}(q) \text { for any } \quad 1 \leq k \leq N .
$$

Since we also have

$$
\frac{\partial \Omega(q)}{\partial \sigma_{k}}=\frac{\partial}{\partial \sigma_{k}}\left(\eta_{0}+\tau_{1}(q) \eta_{1}+\tau_{2}(q) \eta_{2}+\cdots \tau_{N}(q) \eta_{N}+g_{0}(q)\right)
$$

we get $\left[\frac{\partial \tau_{i}(q)}{\partial \sigma_{j}}\right]_{1 \leq i, j \leq N}=\mathrm{I}_{N}$. This shows that $\tau_{*}$ is an isomorphism, as $\left\{\frac{\partial}{\partial \sigma_{1}}, \cdots, \frac{\partial}{\partial \sigma_{N}}\right\}$ is a basis for $T_{q}^{1,0}(\mathcal{T})$.

By Proposition 3.11, the holomorphic map $\tau: \mathcal{T} \rightarrow \mathbb{C}^{N}$ defines local coordinate map around each point $q \in \mathcal{T}$. In particular, the map $\tau$ itself gives a global holomorphic coordinate for $\mathcal{T}$. Thus the transition maps are all identity maps. Therefore,

ThEOREM 3.12. The global holomorphic coordinate map $\tau: \mathcal{T} \rightarrow \mathbb{C}^{N}$ defines a holomorphic affine structure on $\mathcal{T}$.

REMARK 3.13. This affine structure on $\mathcal{T}$ depends on the choice of the base point $p$. Affine structures on $\mathcal{T}$ defined in this ways by fixing different base point may not be compatible with each other.

4. Hodge metric completion of the Teichmüller space with level structure. In Section 4.1, given $m \geq 3$, we introduce the Hodge metric completion $\mathcal{T}^{H}$ of the Teichmüller space with level $m$ structure, which is the universal cover of $\mathcal{Z}_{m}^{m}$, where $\mathcal{Z}_{m}^{H}$ is the completion space of the smooth moduli space $\mathcal{Z}_{m}$ with respect to the Hodge metric. We denote the lifting maps $i_{m}: \mathcal{T} \rightarrow \mathcal{T}_{m}^{H}, \Phi_{m}^{H}: \mathcal{T}_{m}^{H} \rightarrow D$ and $\mathcal{T}_{m}:=i_{m}(\mathcal{T})$. We prove that $\Phi_{m}^{H}$ is a bounded holomorphic map from $\mathcal{T}_{m}^{m}$ to $N_{+} \cap D$. In Section 4.2, we first define the map $\tau_{m}^{H}$ from $\mathcal{T}_{m}^{H}$ to $\mathbb{C}^{N}$ and its restriction $\tau_{m}$ on the submanifold $\mathcal{T}_{m}$. We then show that $\tau_{m}$ is also a local embedding and conclude that $\tau_{m}$ defined a global holomorphic affine structure on $\mathcal{T}_{m}$. Then the affineness of $\tau_{m}$ shows that the extension map $\tau_{m}^{H}$ is also defines a holomorphic affine structure on $\mathcal{T}_{m}^{H}$. In Section 4.3, we prove that $\tau_{m}^{H}$ is an injection by using the Hodge metric completeness and the global holomorphic affine structure on $\mathcal{T}_{m}^{H}$. As a corollary, we show that the holomorphic map $\Phi_{m}^{H}$ is an injection.

4.1. Definitions and basic properties. Recall in Section $2.2, \mathcal{Z}_{m}$ from [20] is the smooth moduli space of polarized Calabi-Yau manifolds with level $m$ structure. We defined the Teichmüller space $\mathcal{T}$ to be the universal cover of $\mathcal{Z}_{m}$. In particular, we have proved that the definition of $\mathcal{T}$ does not depend on the choice of level structures.

By Viehweg's work in [23], we know that $\mathcal{Z}_{m}$ is quasi-projective and that we can find a smooth projective compactification $\overline{\mathcal{Z}}_{m}$ such that $\mathcal{Z}_{m}$ is open in $\overline{\mathcal{Z}}_{m}$ and the complement $\overline{\mathcal{Z}}_{m} \backslash \mathcal{Z}_{m}$ is a divisor of normal crossing. Therefore, $\mathcal{Z}_{m}$ is dense and open 
in $\overline{\mathcal{Z}}_{m}$ where the complex codimension of the complement $\overline{\mathcal{Z}}_{m} \backslash \mathcal{Z}_{m}$ is at least one. Moreover, as $\overline{\mathcal{Z}}_{m}$ a compact space, it is a complete space.

Recall at the end of Section 2.3, we pointed out that there are induced Hodge metrics on $\mathcal{Z}_{m}$. Let us now take $\mathcal{Z}_{m}^{H}$ to be the Hodge metric completion of $\mathcal{Z}_{m}$. Then $\mathcal{Z}_{m}^{H}$ is the smallest complete space with respect to the Hodge metric that contains $\mathcal{Z}_{m}$. Although the compact space $\overline{\mathcal{Z}}_{m}$ may not be unique, the Hodge metric completion space $\mathcal{Z}_{m}^{H}$ is unique up to isometry. In particular, $\mathcal{Z}_{m}^{H} \subseteq \overline{\mathcal{Z}}_{m}$ and the complex codimension of the complement $\mathcal{Z}_{m}^{H} \backslash \mathcal{Z}_{m}$ is at least one. Given a fixed reference point $p$, then any point in $\mathcal{Z}_{m}^{H}$ that is of Hodge finite distance from $p$ has a neighborhood $U \subseteq \overline{\mathcal{Z}}_{m}$, which is also at finite Hodge distance from the reference point $p$. As $\overline{\mathcal{Z}}_{m} \backslash \mathcal{Z}_{m}$ is at least of complex codimension one in $\overline{\mathcal{Z}}_{m}$ and that any two points in $U$ have finite Hodge distance, we have $U \subseteq \mathcal{Z}_{m}^{H}$. This implies that $\mathcal{Z}_{m}^{H}$ is an open submanifold of $\overline{\mathcal{Z}}_{m}$. In particular, any two points in $\mathcal{Z}_{m}^{H}$ are of Hodge finite distance. We summarize the above observations in the following lemma.

Lemma 4.1. The Hodge metric completion $\mathcal{Z}_{m}^{H}$ is a dense and open smooth submanifold in $\overline{\mathcal{Z}}_{m}$ and the complex codimenison of $\mathcal{Z}_{m}^{H} \backslash \mathcal{Z}_{m}$ is at least one.

Let $\mathcal{T}_{m}^{H}$ be the universal cover of $\mathcal{Z}_{m}^{H}$. Thus $\mathcal{T}_{m}^{H}$ is a connected and simply connected complete smooth complex manifold with respect to the Hodge metric. We will call $\mathcal{T}_{m}^{H}$ the Hodge metric completion space with level $m$ structure of $\mathcal{T}$, or simply the Hodge metric completion space. We denote the universal covering map by $\pi_{m}^{H}$ : $\mathcal{T}_{m}^{H} \rightarrow \mathcal{Z}_{m}^{H}$. Since $\mathcal{Z}_{m}^{H}$ is the Hodge metric completion of $\mathcal{Z}_{m}$, there exists the natural continuous extension map $\Phi_{\mathcal{Z}_{m}}^{H}: \mathcal{Z}_{m}^{H} \rightarrow D / \Gamma$. Moreover, recall that the Teichmüller space $\mathcal{T}$ is the universal cover of the moduli space $\mathcal{Z}_{m}$ with the universal covering map denoted by $\pi_{m}: \mathcal{T} \rightarrow \mathcal{Z}_{m}$. Thus we have the following commutative diagram

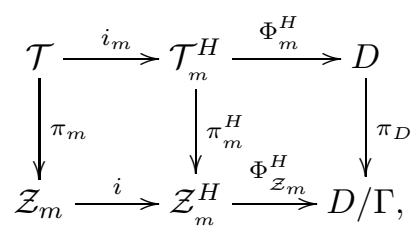

where $i$ is the inclusion map, $i_{m}$ is a lifting map of $i \circ \pi_{m}, \pi_{D}$ is the covering map and $\Phi_{m}^{H}$ is a lifting map of $\Phi_{\mathcal{Z}_{m}}^{H} \circ \pi_{m}^{H}$. In particular, $\Phi_{m}^{H}$ is a continuous map from $\mathcal{T}_{m}^{H}$ to $D$. One may notice that the lifting maps $i_{\mathcal{T}}$ and $\Phi_{m}^{H}$ are not unique, but it is not hard to show implies that there exists a suitable choice of $i_{m}$ and $\Phi_{m}^{H}$ such that $\Phi=\Phi_{m}^{H} \circ i_{m}$. We refer the reader to the appendix of [1] for the proof of this simple fact. We will fix the choice of $i_{m}$ and $\Phi_{m}^{H}$ such that $\Phi=\Phi_{m}^{H} \circ i_{m}$ in the rest of the paper.

REMARK 4.2. Unless otherwise pointed out, when we mention a complete space in this paper, the completeness is always with respect to the Hodge metric.

Let us consider $\mathcal{T}_{m}:=i_{m}(\mathcal{T})$, which is connected as $\mathcal{T}$ is. Then we have the following result, the proof of which is provided in [1].

Proposition 4.3. The image $\mathcal{T}_{m}:=i_{m}(\mathcal{T})$ equals to the preimage $\left(\pi_{m}^{H}\right)^{-1}\left(\mathcal{Z}_{m}\right)$.

One notices that the restriction map $\Phi_{m}$ is holomorphic. Indeed, we know that $i_{m}: \mathcal{T} \rightarrow \mathcal{T}_{m}$ is the lifting of $i \circ \pi_{m}$ and $\left.\pi_{m}^{H}\right|_{\mathcal{T}_{m}}: \mathcal{T}_{m} \rightarrow \mathcal{Z}_{m}$ is a holomorphic covering map, thus $i_{m}$ is also holomorphic. Since $\Phi=\Phi_{m} \circ i_{m}$ with both $\Phi, i_{m}$ holomorphic 
and $i_{m}$ locally invertible, we can conclude that $\Phi_{m}: \mathcal{T}_{m} \rightarrow D$ is a holomorphic map. Moreover, we have $\Phi_{m}\left(\mathcal{T}_{m}\right)=\Phi_{m}\left(i_{m}(\mathcal{T})\right)=\Phi(\mathcal{T})$ as $\Phi=i_{m} \circ \Phi_{m}$. In particular, as $\Phi: \mathcal{T} \rightarrow N_{+} \cap D$ is bounded, we get that $\Phi_{m}: \mathcal{T} \rightarrow N_{+} \cap D$ is bounded in $N_{+}$ with the Euclidean metric. Thus $\Phi_{m}^{H}$ is also bounded. Therefore applying Riemann extension theorem, we get

Proposition 4.4. The map $\Phi_{m}^{H}$ is a bounded holomorphic map from $\mathcal{T}_{m}^{H}$ to $N_{+} \cap D$.

Proof. According to the above discussion, we know that the complement $\mathcal{T}_{m}^{H} \backslash \mathcal{T}_{m}$ is the pre-image of $\mathcal{Z}_{m}^{H} \backslash \mathcal{Z}_{m}$ of the covering map $\pi_{m}^{H}$. So $\mathcal{T}_{m}^{H} \backslash \mathcal{T}_{m}$ is a analytic subvariety of $\mathcal{T}_{m}^{H}$, with complex codimension at least one and $\Phi_{m}: \mathcal{T}_{m} \rightarrow N_{+} \cap D$ is a bounded holomorphic map. Therefore, simply applying the Riemman extension theorem to the holomorphic map $\Phi_{m}: \mathcal{T}_{m} \rightarrow N_{+} \cap D$, we conclude that there exists a holomorphic map $\Phi_{m}^{\prime}: \mathcal{T}_{m}^{H} \rightarrow N_{+} \cap D$ such that $\left.\Phi_{m}^{\prime}\right|_{\mathcal{T}_{m}}=\Phi_{m}$. We know that both $\Phi_{m}^{H}$ and $\Phi_{m}^{\prime}$ are continuous maps defined on $\mathcal{T}_{m}^{H}$ that agree on the dense subset $\mathcal{T}_{m}$. Therefore, they must agree on the whole $\mathcal{T}_{m}^{H}$, that is, $\Phi_{m}^{H}=\Phi_{m}^{\prime}$ on $\mathcal{T}_{m}^{H}$. Therefore, $\Phi_{m}^{H}: \mathcal{T}_{m}^{H} \rightarrow N_{+} \cap D$ is a bounded holomorphic map.

4.2. Holomorphic affine structure on the Hodge metric completion space . In this section, we still fix the base point $\Phi(p) \in D$ with $p \in \mathcal{T}$ and an adapted basis $\left(\eta_{0}, \cdots, \eta_{m-1}\right)$ for the Hodge decomposition of $\Phi(p)$. We defined the global coordinate map $\tau: \mathcal{T} \rightarrow \mathbb{C}^{N}$, which is a holomorphic affine local embedding. Let us now define

$$
\tau_{m}:=P \circ \Phi_{m}: \mathcal{T}_{m} \rightarrow \mathbb{C}^{N}
$$

where $P: N_{+} \cap D \rightarrow \mathbb{C}^{N}$ is the projection map defined in (14). Then as $\Phi=\Phi_{m} \circ i_{m}$, we get $\tau=P \circ \Phi=P \circ \Phi_{m} \circ i_{m}=\tau_{m} \circ i_{m}$.

In the following lemma, we will crucially use the fact that the holomorphic map $\tau: \mathcal{T} \rightarrow \mathbb{C}^{N}$ is a local embedding.

LEMMA 4.5. The holomorphic map $\tau_{m}: \mathcal{T} \rightarrow \mathbb{C}^{N}$ is a local embedding. In particular, $\tau_{m}: \mathcal{T}_{m} \rightarrow \mathbb{C}^{N}$ defines a global holomorphic affine structure on $\mathcal{T}_{m}$.

Proof. Since $i \circ \pi_{m}=\pi_{m}^{H} \circ i_{m}$ with $i: \mathcal{Z}_{m} \rightarrow \mathcal{Z}_{m}^{H}$ the natural inclusion map and $\pi_{m}, \pi_{m}^{H}$ both universal covering maps, $i_{m}$ is a lifting of the inclusion map. Thus $i_{m}$ is locally biholomorphic. On the other hand, we showed that $\tau$ is a local embedding. We may choose an open cover $\left\{U_{\alpha}\right\}_{\alpha \in \Lambda}$ of $\mathcal{T}_{m}$ such that for each $U_{\alpha} \subseteq \mathcal{T}_{m}, i_{m}$ is biholomorphic on $U_{\alpha}$ and thus the inverse $\left(i_{m}\right)^{-1}$ is also an embedding on $U_{\alpha}$. Obviously we may also assume that $\tau$ is an embedding on $\left(i_{m}\right)^{-1}\left(U_{\alpha}\right)$. In particular, the relation $\tau=\tau_{m} \circ i_{m}$ implies that $\left.\tau_{m}\right|_{U_{\alpha}}=\left.\tau \circ\left(i_{m}\right)^{-1}\right|_{U_{\alpha}}$ is also an embedding on $U_{\alpha}$. In this way, we showed $\tau_{m}$ is a local embedding on $\mathcal{T}_{m}$. Therefore, since $\operatorname{dim}_{\mathbb{C}} \mathcal{T}_{m}=N, \tau_{m}: \mathcal{T}_{m} \rightarrow \mathbb{C}^{N}$ defines a local coordinate map around each point in $\mathcal{T}_{m}$. In particular, the map $\tau_{m}$ itself gives a global holomorphic coordinate for $\mathcal{T}_{m}$. Thus the transition maps are all identity maps. Therefore, $\tau_{m}: \mathcal{T}_{m} \rightarrow \mathbb{C}^{N}$ defines a global holomorphic affine structure on $\mathcal{T}_{m}$.

Let us define $\tau_{m}^{H}:=P \circ \Phi_{m}^{H}: \mathcal{T}_{m}^{H} \rightarrow \mathbb{C}^{N}$, where $P: N_{+} \cap D \rightarrow \mathbb{C}^{N}$ is still the projection map defined in (14). Then we easily see that $\left.\tau_{m}^{H}\right|_{\mathcal{T}_{m}}=\tau_{m}$. We also have the following, ding.

LEMMA 4.6. The holomorphic map $\tau_{m}^{H}: \mathcal{T}_{m}^{H} \rightarrow \mathbb{C}^{N} \cong H_{p}^{n-1,1}$ is a local embed- 
Proof. The proof uses mainly the affineness of $\tau_{m}: \mathcal{T}_{m} \rightarrow \mathbb{C}^{N} \cong H_{p}^{n-1,1}$. By Proposition 4.3, we know that $\mathcal{T}_{m}$ is dense and open in $\mathcal{T}_{m}^{H}$. Thus for any point $q \in$ $\mathcal{T}_{m}^{H}$, there exists $\left\{q_{k}\right\}_{k=1}^{\infty} \subseteq \mathcal{T}_{m}$ such that $\lim _{k \rightarrow \infty} q_{k}=q$. Because $\tau_{m}^{H}(q) \in H_{p}^{n-1,1}$, we can take a neighborhood $W \subseteq H_{p}^{n-1,1}$ of $\tau_{m}^{H}(q)$ with $W \subseteq \tau_{m}^{H}\left(\mathcal{T}_{m}^{H}\right)$.

Consider the projection map $P: N_{+} \rightarrow \mathbb{C}^{N}$ with $P(F)=F^{(1,0)}$ the $(1,0)$ block of the matrix $F$, and the decomposition of the holomorphic tangent bundle

$$
T^{1,0} N_{+}=\bigoplus_{0 \leq l \leq k \leq n} \operatorname{Hom}\left(F^{k} / F^{k+1}, F^{l} / F^{l+1}\right) .
$$

In particular, the subtangent bundle $\operatorname{Hom}\left(F^{n}, F^{n-1} / F^{n}\right)$ over $N_{+}$is isomorphic to the pull-back bundle $P^{*}\left(T^{1,0} \mathbb{C}^{N}\right)$ of the holomorphic tangent bundle of $\mathbb{C}^{N}$ through the projection $P$. On the other hand, the holomorphic tangent bundle of $\mathcal{T}_{m}$ is also isomorphic to the holomorphic bundle $\operatorname{Hom}\left(F^{n}, F^{n-1} / F^{n}\right)$, where $F^{n}$ and $F^{n-1}$ are pull-back bundles on $\mathcal{T}_{m}$ via $\Phi_{m}$ from $N_{+} \cap D$. Since the holomorphic map $\tau_{m}=P \circ \Phi_{m}$ is a composition of $P$ and $\Phi_{m}$, the pull-back bundle of $T^{1,0} W$ through $\tau_{m}$ is also isomorphic to the tangent bundle of $\mathcal{T}_{m}$.

Now with the fixed adapted basis $\left\{\eta_{1}, \cdots, \eta_{N}\right\}$, one has a standard coordinate $\left(z_{1}, \cdots, z_{N}\right)$ on $\mathbb{C}^{N} \cong H_{p}^{n-1,1}$ such that each point in $\mathbb{C}^{N} \cong H_{p}^{n-1,1}$ is of the form $z_{1} \eta_{1}+\cdots+z_{N} \eta_{N}$. Let us choose one special trivialization of

$$
T^{1,0} W \cong \operatorname{Hom}\left(F_{p}^{n}, F_{p}^{n-1} / F_{p}^{n}\right) \times W
$$

by the standard global holomorphic frame $\left(\Lambda_{1}, \cdots, \Lambda_{N}\right)=\left(\partial / \partial z_{1}, \cdots, \partial / \partial z_{N}\right)$ on $T^{1,0} W$. Under this trivialization, we can identify $T_{o}^{1,0} W$ with $\operatorname{Hom}\left(F_{p}^{n}, F_{p}^{n-1} / F_{p}^{n}\right)$ for any $o \in W$. Then $\left(\Lambda_{1}, \cdots, \Lambda_{N}\right)$ are parallel sections with respect to the trivial affine connection on $T^{1,0} W$. Let $U_{q} \subseteq\left(\tau_{m}^{H}\right)^{-1}(W)$ be a neighborhood of $q$ and let $U=U_{q} \cap \mathcal{T}_{m}$. Then the pull back sections $\left(\tau_{m}^{H}\right)^{*}\left(\Lambda_{1}, \cdots, \Lambda_{N}\right): U_{q} \rightarrow T^{1,0} U_{q}$ are tangent vectors of $U_{q}$, we denote them by $\left(\mu_{1}^{H}, \cdots, \mu_{N}^{H}\right)$ for convenience.

According to the proof of Lemma 4.5, we know that the restriction map $\tau_{m}$ is a local embedding. Therefore the tangent map $\left(\tau_{m}\right)_{*}: T_{q^{\prime}}^{1,0} U \rightarrow T_{o}^{1,0} \mathrm{~W}$ is an isomorphism, for any $q^{\prime} \in U$ and $o=\tau_{m}\left(q^{\prime}\right)$. Moreover, since $\tau_{m}$ is a holomorphic affine map, the holomorphic sections $\left(\mu_{1}, \cdots, \mu_{N}\right):=\left.\left(\mu_{1}^{H}, \cdots, \mu_{N}^{H}\right)\right|_{U}$ form a holomorphic parallel frame for $T^{1,0} U$. Under the parallel frames $\left(\mu_{1}, \cdots, \mu_{N}\right)$ and $\left(\Lambda_{1}, \cdots, \Lambda_{N}\right)$, there exists a nonsingular matrix function $A\left(q^{\prime}\right)=\left(a_{i j}\left(q^{\prime}\right)\right)_{1 \leq i \leq N, 1 \leq j \leq N}$, such that the tangent map $\left(\tau_{m}\right)_{*}$ is given by

$$
\left(\tau_{m}\right)_{*}\left(\mu_{1}, \cdots, \mu_{N}\right)\left(q^{\prime}\right)=\left(\Lambda_{1}(o), \cdots, \Lambda_{N}(o)\right) A\left(q^{\prime}\right), \quad \text { with } q^{\prime} \in U \text { and } o=\tau_{m}\left(q^{\prime}\right) \in D .
$$

Moreover, since $\left(\Lambda_{1}, \cdots, \Lambda_{N}\right)$ and $\left(\mu_{1}, \cdots, \mu_{N}\right)$ are parallel frames for $T^{1,0} W$ and $T^{1,0} U$ respectively and $\tau_{m}$ is a holomorphic affine map, the matrix $A\left(q^{\prime}\right)=A$ is actually a constant nonsingular matrix for all $q^{\prime} \in U$. In particular, for each $q_{k} \in U$, we have $\left(\left(\tau_{m}\right)_{*} \mu_{1}, \cdots,\left(\tau_{m}\right)_{*} \mu_{N}\right)\left(q_{k}\right)=\left(\Lambda_{1}\left(o_{k}\right), \cdots, \Lambda_{N}\left(o_{k}\right)\right) A$, where $o_{k}=\tau_{m}\left(q_{k}\right)$. Because the tangent map $\left(\tau_{m}^{H}\right)_{*}: T^{1,0} U_{q} \rightarrow T^{1,0} W$ is a continuous map, we have that

$$
\begin{gathered}
\left(\tau_{m}^{H}\right)_{*}\left(\mu_{1}^{H}(q), \cdots, \mu_{N}^{H}(q)\right)=\lim _{k \rightarrow \infty}\left(\tau_{m}\right)_{*}\left(\mu_{1}\left(q_{k}\right), \cdots, \mu_{N}\left(q_{k}\right)\right)=\lim _{k \rightarrow \infty}\left(\Lambda_{1}\left(o_{k}\right), \cdots, \Lambda_{N}\left(o_{k}\right)\right) A \\
=\left(\Lambda_{1}(\bar{o}), \cdots, \Lambda_{N}(\bar{o})\right) A, \quad \text { where } \quad o_{k}=\tau_{m}\left(q_{k}\right) \text { and } \bar{o}=\tau_{m}^{H}(q) .
\end{gathered}
$$

As $\left(\Lambda_{1}(\bar{o}), \cdots, \Lambda_{N}(\bar{o})\right)$ forms a basis for $T_{\bar{o}}^{1,0} W=\operatorname{Hom}\left(F_{p}^{n}, F_{p}^{n-1} / F_{p}^{n}\right)$ and $A$ is nonsingular, we can conclude that $\left(\tau_{m}^{H}\right)_{*}$ is an isomorphism from $T_{q}^{1,0} U_{q}$ to $T_{\bar{o}}^{1,0} W$. This shows that $\tau_{m}^{H}: \mathcal{T}_{m}^{H} \rightarrow \mathbb{C}^{N} \cong H_{p}^{n-1,1}$ is a local embedding. 
ThEOREM 4.7. The holomorphic map $\tau_{m}^{H}: \mathcal{T}_{m}^{H} \rightarrow \mathbb{C}^{N}$ defines a global holomorphic affine structure on $\mathcal{T}_{m}^{H}$.

Proof. Since $\tau_{m}^{H}: \mathcal{T}_{m}^{H} \rightarrow \mathbb{C}^{N}$ is a local embedding and $\operatorname{dim} \mathcal{T}_{m}^{H}=N$, thus the same arguments as the proof of Lemma 4.5 can be applied to conclude $\tau_{m}^{H}$ defines a global holomorphic affine structure on $\mathcal{T}_{m}^{H}$.

It is important to note that the flat connections which correspond to the global holomorphic affine structures on $\mathcal{T}$, on $\mathcal{T}_{m}$ or on $\mathcal{T}_{m}^{H}$ are in general not compatible to the corresponding Hodge metrics on them.

\subsection{Injectivity of the period map on the Hodge metric completion space.}

THEOREM 4.8. For any $m \geq 3$, the holomorphic map $\tau_{m}^{H}: \mathcal{T}_{m}^{H} \rightarrow \mathbb{C}^{N}$ is an injection.

To prove this theorem, we will first prove the following elementary lemma, where we mainly use the completeness with the Hodge metric, the holomorphic affine structure on $\mathcal{T}_{m}^{H}$, the affineness of $\tau_{m}^{H}$, and the properties of Hodge metric. We remark that as $\mathcal{T}_{m}^{m}$ is a complex affine manifold, we have the notion of straight lines in it with respect to the affine structure.

LEMMA 4.9. For any two points in $\mathcal{T}_{m}^{H}$, there is a straight line in $\mathcal{T}_{m}^{H}$ connecting them.

Proof. Let $p$ be an arbitrary point in $\mathcal{T}_{m}^{H}$, and let $S \subseteq \mathcal{T}_{m}^{H}$ be the collection of points that can be connected to $p$ by straight lines in $\mathcal{T}_{m}^{\bar{H}}$. We need to show that $S=\mathcal{T}_{m}^{H}$.

We first show that $S$ is a closed set. Let $\left\{q_{i}\right\}_{i=1}^{\infty} \subseteq S$ be a Cauchy sequence with respect to the Hodge metric. Then for each $i$ we have the straight line $l_{i}$ connecting $p$ and $q_{i}$ such that $l_{i}(0)=p, l_{i}\left(T_{i}\right)=q_{i}$ for some $T_{i} \geq 0$ and $v_{i}:=\frac{\partial}{\partial t} l_{i}(0)$ a unit vector with respect to the Euclidean metric on $n_{+}$. We can view these straight lines $l_{i}:\left[0, T_{i}\right] \rightarrow \mathcal{T}_{m}^{H}$ as the solutions of the affine geodesic equations $l_{i}^{\prime \prime}(t)=0$ with initial conditions $v_{i}:=\frac{\partial}{\partial t} l_{i}(0)$ and $l_{i}(0)=p$ in particular $T_{i}=d_{E}\left(p, q_{i}\right)$ is the Euclidean distance between $p$ and $q_{i}$. It is well-known that solutions of these geodesic equations analytically depend on their initial data.

Proposition 4.4 showed that $\Phi_{m}^{H}: \mathcal{T}_{m}^{H} \rightarrow N_{+} \cap D$ is a bounded map, which implies that the image of $\Phi_{m}^{H}$ is bounded with respect to the Euclidean metric on $N_{+}$. Because a linear projection will map a bounded set to a bounded set, we have that the image of $\tau_{m}^{H}=P \circ \Phi_{m}^{H}$ is also bounded in $\mathbb{C}^{N}$ with respect to the Euclidean metric on $\mathbb{C}^{N}$. Passing to a subsequence, we may therefore assume that $\left\{T_{i}\right\}$ and $\left\{v_{i}\right\}$ converge, with $\lim _{i \rightarrow \infty} T_{i}=T_{\infty}$ and $\lim _{i \rightarrow \infty} v_{i}=v_{\infty}$, respectively. Let $l_{\infty}(t)$ be the local solution of the affine geodesic equation with initial conditions $\frac{\partial}{\partial t} l_{\infty}(0)=v_{\infty}$ and $l_{\infty}(0)=p$. We claim that the solution $l_{\infty}(t)$ exists for $t \in\left[0, T_{\infty}\right]$. Consider the set

$$
E_{\infty}:=\left\{a: l_{\infty}(t) \text { exists for } t \in[0, a)\right\} .
$$

If $E_{\infty}$ is unbounded above, then the claim clearly holds. Otherwise, we let $a_{\infty}=$ $\sup E_{\infty}$, and our goal is to show $a_{\infty}>T_{\infty}$. Suppose towards a contradiction that $a_{\infty} \leq T_{\infty}$. We then define the sequence $\left\{a_{k}\right\}_{k=1}^{\infty}$ so that $a_{k} / T_{k}=a_{\infty} / T_{\infty}$. We have $a_{k} \leq T_{k}$ and $\lim _{k \rightarrow \infty} a_{k}=a_{\infty}$. Using the continuous dependence of solutions of the geodesic equation on initial data, we conclude that the sequence $\left\{l_{k}\left(a_{k}\right)\right\}_{k=1}^{\infty}$ is 
a Cauchy sequence. As $\mathcal{T}_{m}^{H}$ is a complete space, the sequence $\left\{l_{k}\left(a_{k}\right)\right\}_{k=1}^{\infty}$ converges to some $q^{\prime} \in \mathcal{T}_{m}^{H}$. Let us define $l_{\infty}\left(a_{\infty}\right):=q^{\prime}$. Then the solution $l_{\infty}(t)$ exists for $t \in\left[0, a_{\infty}\right]$. On the other hand, as $\mathcal{T}_{m}^{H}$ is a smooth manifold, we have that $q^{\prime}$ is an inner point of $\mathcal{T}_{m}^{H}$. Thus the affine geodesic equation has a local solution at $q^{\prime}$ which extends the geodesic $l_{\infty}$. That is, there exists $\epsilon>0$ such that the solution $l_{\infty}(t)$ exists for $t \in\left[0, a_{\infty}+\epsilon\right)$. This contradicts the fact that $a_{\infty}$ is an upper bound of $E_{\infty}$. We have therefore proven that $l_{\infty}(t)$ exists for $t \in\left[0, T_{\infty}\right]$.

Using the continuous dependence of solutions of the affine geodesic equations on the initial data again, we get

$$
l_{\infty}\left(T_{\infty}\right)=\lim _{k \rightarrow \infty} l_{k}\left(T_{k}\right)=\lim _{k \rightarrow \infty} q_{k}=q_{\infty}
$$

This means the limit point $q_{\infty} \in S$, and hence $S$ is a closed set.

Let us now show that $S$ is an open set. Let $q \in S$. Then there exists a straight line $l$ connecting $p$ and $q$. For each point $x \in l$ there exists an open neighborhood $U_{x} \subseteq \mathcal{T}_{m}^{H}$ with diameter $2 r_{x}$. The collection of $\left\{U_{x}\right\}_{x \in l}$ forms an open cover of $l$. But $l$ is a compact set, so there is a finite subcover $\left\{U_{x_{i}}\right\}_{i=1}^{K}$ of $l$. Then the straight line $l$ is covered by a cylinder $C_{r}$ in $\mathcal{T}_{m}^{H}$ of radius $r=\min \left\{r_{x_{i}}: 1 \leq i \leq K\right\}$. As $C_{r}$ is a convex set, each point in $C_{r}$ can be connected to $p$ by a straight line. Therefore we have found an open neighborhood $C_{r}$ of $q \in S$ such that $C_{r} \subseteq S$, which implies that $S$ is an open set.

As $S$ is a non-empty, open and closed subset in the connected space $\mathcal{T}_{m}^{H}$, we conclude that $S=\mathcal{T}_{m}^{H}$, as we desired.

Proof of Theorem 4.8. Let $p, q \in \mathcal{T}_{m}^{H}$ be two different points. Then Lemma 4.9 implies that there is a straight line $l \subseteq \mathcal{T}_{m}^{m}$ connecting $p$ and $q$. Since $\tau_{m}^{H}: \mathcal{T}_{m}^{H} \rightarrow \mathbb{C}^{N}$ is affine, the restriction $\left.\tau_{m}^{H}\right|_{l}$ is a linear map. Suppose towards a contradiction that $\tau_{m}^{H}(p)=\tau_{m}^{H}(q) \in \mathbb{C}^{N}$. Then the restriction of $\tau_{m}^{H}$ to the straight line $l$ is a constant map as $\left.\tau_{m}^{H}\right|_{l}$ is linear. By Lemma 4.6, we know that $\tau_{m}^{H}: \mathcal{T}_{m}^{H} \rightarrow \mathbb{C}^{N}$ is locally injective. Therefore, we may take $U_{p}$ to be a neighborhood of $p$ in $\mathcal{T}_{m}^{H}$ such that $\tau_{m}^{H}: U_{p} \rightarrow \mathbb{C}^{N}$ is injective. However, the intersection of $U_{p}$ and $l$ contains infinitely many points, but the restriction of $\tau_{m}^{H}$ to $U_{p} \cap l$ is a constant map. This contradicts the fact that when we restrict $\tau_{m}^{H}$ to $U_{p} \cap l, \tau_{m}^{H}$ is an injective map. Thus $\tau_{m}^{H}(p) \neq \tau_{m}^{H}(q)$ if $p \neq q \in \mathcal{T}_{m}^{H}$.

Since $\tau_{m}^{H}=P \circ \Phi_{m}^{H}$, where $P$ a the projection map and $\tau_{m}^{H}$ is injective and $\Phi_{m}^{H}$ a bounded map, we get

Corollary 4.10. The completion space $\mathcal{T}_{m}^{H}$ is a bounded domain in $\mathbb{C}^{N}$.

Corollary 4.11. The holomorphic map $\Phi_{m}^{H}: \mathcal{T}_{m}^{H} \rightarrow N_{+} \cap D$ is also an injection.

5. Hodge metric completion of the Teichmüller space and domain of holomorphy. In this section, We will review the proof of one of our main results in this section, which is Theorem 5.3. In this section, we define the completion space $\mathcal{T}^{H}$ by $\mathcal{T}^{H}=\mathcal{T}_{m}^{H}$, and the extended period map $\Phi^{H}$ by $\Phi^{H}=\Phi_{m}^{H}$ for any $m \geq 3$ after proving that $\mathcal{T}_{m}^{H}$ doesn't depend on the choice of the level $m$. Therefore $\overline{\mathcal{T}}^{H}$ is a complex affine manifold and that $\Phi^{H}$ is a holomorphic injection. We then prove the main result Theorem 5.3, which asserts that $\mathcal{T}^{H}$ is the completion space of $\mathcal{T}$ with respect to the Hodge metric and it is a bounded domain of holomorphy in $\mathbb{C}^{N}$. As a direct corollary, we get the global Torelli theorem of the period map from the Teichmüller space to the period domain. 
For any two integers $m, m^{\prime} \geq 3$, let $\mathcal{Z}_{m}$ and $\mathcal{Z}_{m^{\prime}}$ be the smooth quasi-projective manifolds as in Theorem 2.3 and $\mathcal{Z}_{m}^{H}$ and $\mathcal{Z}_{m^{\prime}}^{H}$ their Hodge metric completions. Let $\mathcal{T}_{m}^{H}$ and $\mathcal{T}_{m^{\prime}}^{H}$ be the universal cover spaces of $\mathcal{Z}_{m}^{H}$ and $\mathcal{Z}_{m^{\prime}}^{H}$ respectively, then we have the following.

Proposition 5.1. The complete complex manifolds $\mathcal{T}_{m}^{H}$ and $\mathcal{T}_{m^{\prime}}^{H}$ are biholomorphic to each other.

Proposition 5.1 shows that $\mathcal{T}_{m}^{H}$ doesn't depend on the choice of the level $m$ structure, and it allows us to give the following definition.

Definition 5.2. We define the complete complex manifold $\mathcal{T}^{H}=\mathcal{T}^{H}$, the holomorphic map $i_{\mathcal{T}}: \mathcal{T} \rightarrow \mathcal{T}^{H}$ by $i_{\mathcal{T}}=i_{m}$, and the extended period map $\Phi^{H}: \mathcal{T}^{H} \rightarrow D$ by $\Phi^{H}=\Phi_{m}^{H}$ for any $m \geq 3$. In particular, with these new notations, we have the commutative diagram

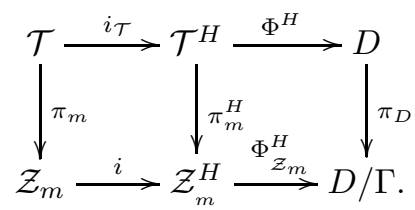

THEOREM 5.3. The complex manifold $\mathcal{T}^{H}$, which is a complex affine manifold and can be embedded into $\mathbb{C}^{N}$, is the completion space of $\mathcal{T}$ with respect to the Hodge metric. Moreover, the extended period map $\Phi^{H}: \mathcal{T}^{H} \rightarrow N_{+} \cap D$ is a holomorphic injection.

Proof. By the definition of $\mathcal{T}^{H}$, Theorem 4.7, and Corollary 4.10, it is easy to see that $\mathcal{T}_{m}^{H}$ is a complex affine manifold, which can be embedded into $\mathbb{C}^{N}$. It is also not hard to see that the injectivity of $\Phi^{H}$ follows from Corollary 4.11 by the definition of $\Phi^{H}$. Now to prove this theorem, it is sufficient to show that $i_{\mathcal{T}}: \mathcal{T} \rightarrow \mathcal{T}^{H}$ is an embedding, which is given in the following lemma.

LEMma 5.4. The map $i_{\mathcal{T}}: \mathcal{T} \rightarrow \mathcal{T}^{H}$ is an embedding.

Proof. On one hand, define $\mathcal{T}_{0}$ to be $\mathcal{T}_{0}=\mathcal{T}_{m}$ for any $m \geq 3$, as $\mathcal{T}_{m}$ doesn't depend on the choice of $m$ according to the proof of Proposition 5.1. Since $\mathcal{T}_{0}=\left(\pi_{m}^{H}\right)^{-1}\left(\mathcal{Z}_{m}\right)$, $\pi_{m}^{H}: \mathcal{T}_{0} \rightarrow \mathcal{Z}_{m}$ is a covering map. Thus the universal property of the universal covering map $\pi_{m}: \mathcal{T} \rightarrow \mathcal{Z}_{m}$ and that $\pi_{m}=\left.\pi_{m}^{H}\right|_{\mathcal{T}_{0}} \circ i_{\mathcal{T}}$ imply that $i_{\mathcal{T}}: \mathcal{T} \rightarrow \mathcal{T}_{0}$ is also a covering map and that the fundamental group of $\mathcal{T}_{0}$ is a subgroup of the fundamental group of $\mathcal{Z}_{m}$, that is, $\pi_{1}\left(\mathcal{T}_{0}\right) \subseteq \pi_{1}\left(\mathcal{Z}_{m}\right)$, for any $m \geq 3$.

On the other hand, let $\left\{m_{k}\right\}_{k=1}^{\infty}$ be a sequence of positive integers such that $m_{k}<m_{k+1}$ and $m_{k} \mid m_{k+1}$ for each $k \geq 1$. Then there is a natural covering map from $\mathcal{Z}_{m_{k+1}}$ to $\mathcal{Z}_{m_{k}}$ for each $k$. In fact, because each point in $\mathcal{Z}_{m_{k+1}}$ is a polarized Calabi-Yau manifold with a basis $\gamma_{m_{k+1}}$ for the space $\left(H_{n}(M, \mathbb{Z}) /\right.$ Tor $) / m_{k+1}\left(H_{n}(M, \mathbb{Z}) /\right.$ Tor $)$ and $m_{k} \mid m_{k+1}$, then the basis $\gamma_{m_{k+1}}$ induces a basis for the space $\left(H_{n}(M, \mathbb{Z}) /\right.$ Tor $) / m_{k}\left(H_{n}(M, \mathbb{Z}) /\right.$ Tor $)$. Therefore we get a welldefined map $\mathcal{Z}_{m_{k+1}} \rightarrow \mathcal{Z}_{m_{k}}$ by assigning to a polarized Calabi-Yau manifold with the basis $\gamma_{m_{k+1}}$ which is a point in $\mathcal{Z}_{m_{k+1}}$ the same polarized Calabi-Yau manifold with the basis $\left(\gamma_{m_{k+1}}\left(\bmod m_{k}\right)\right) \in\left(H_{n}(M, \mathbb{Z}) /\right.$ Tor $) / m_{k}\left(H_{n}(M, \mathbb{Z}) /\right.$ Tor $)$. Moreover, using the versal properties of both the families $\mathcal{X}_{m_{k+1}} \rightarrow \mathcal{Z}_{m_{k+1}}$ and $\mathcal{X}_{m_{k}} \rightarrow \mathcal{Z}_{m_{k}}$, we can conclude that the map $\mathcal{Z}_{m_{k+1}} \rightarrow \mathcal{Z}_{m_{k}}$ is locally biholomorphic. Therefore, 
$\mathcal{Z}_{m_{k+1}} \rightarrow \mathcal{Z}_{m_{k}}$ is actually a covering map. Thus the fundamental group $\pi_{1}\left(\mathcal{Z}_{m_{k+1}}\right)$ is a subgroup of $\pi_{1}\left(\mathcal{Z}_{m_{k}}\right)$ for each $k$. Hence, the inverse system of fundamental groups

$$
\pi_{1}\left(\mathcal{Z}_{m_{1}}\right) \longleftarrow \pi_{1}\left(\mathcal{Z}_{m_{2}}\right) \longleftarrow \cdots \cdots \pi_{1}\left(\mathcal{Z}_{m_{k}}\right) \longleftarrow \cdots
$$

has an inverse limit, which is the fundamental group of $\mathcal{T}$. Because $\pi_{1}\left(\mathcal{T}_{0}\right) \subseteq \pi_{1}\left(\mathcal{Z}_{m_{k}}\right)$ for any $k$, we have the inclusion $\pi_{1}\left(\mathcal{T}_{0}\right) \subseteq \pi_{1}(\mathcal{T})$. But $\pi_{1}(\mathcal{T})$ is a trivial group since $\mathcal{T}$ is simply connected, thus $\pi_{1}\left(\mathcal{T}_{0}\right)$ is also a trivial group. Therefore the covering map $i_{\mathcal{T}}: \mathcal{T} \rightarrow \mathcal{T}_{0}$ is a one-to-one covering. This shows that $i_{\mathcal{T}}: \mathcal{T} \rightarrow \mathcal{T}^{H}$ is an embedding.

Since $\Phi=\Phi^{H} \circ i_{\mathcal{T}}$ with both $\Phi^{H}$ and $i_{\mathcal{T}}$ embeddings, we get the global Torelli theorem for the period map from the Teichmüller space to the period domain as follows.

Corollary 5.5 (Global Torelli theorem). The period map $\Phi: \mathcal{T} \rightarrow D$ is injective.

As another important consequence, we prove the following property of $\mathcal{T}^{H}$.

THEOREM 5.6. The completion space $\mathcal{T}^{H}$ is a bounded domain of holomorphy in $\mathbb{C}^{N} ;$ thus there exists a complete Kähler-Einstein metric on $\mathcal{T}^{H}$.

We recall that a $\mathcal{C}^{2}$ function $\rho: \Omega \rightarrow \mathbb{R}$ on a domain $\Omega \subseteq \mathbb{C}^{n}$ is plurisubharmornic if and only if its Levi form is positive definite at each point in $\Omega$. Given a domain $\Omega \subseteq \mathbb{C}^{n}$, a function $f: \Omega \rightarrow \mathbb{R}$ is called an exhaustion function if for any $c \in \mathbb{R}$, the set $\{z \in \Omega \mid f(z)<c\}$ is relatively compact in $\Omega$. The following well-known theorem provides a definition for domains of holomorphy. For example, one may refer to [8] for details.

Proposition 5.7. An open set $\Omega \in \mathbb{C}^{n}$ is a domain of holomorphy if and only if there exists a continuous plurisubharmonic function $f: \Omega \rightarrow \mathbb{R}$ such that $f$ is also an exhaustion function.

The following theorem in Section 3.1 of [5] gives us the basic ingredients to construct a plurisubharmoic exhaustion function on $\mathcal{T}^{H}$.

Proposition 5.8. On every manifold $D$, which is dual to a Kähler $C$-space, there exists an exhaustion function $f: D \rightarrow \mathbb{R}$, whose Levi form, restricted to $T_{h}^{1,0}(D)$, is positive definite at every point of $D$.

We remark that in this proposition, in order to show $f$ is an exhaustion function on $D$, Griffiths and Schmid showed that the set $f^{-1}(-\infty, c]$ is compact in $D$ for any $c \in \mathbb{R}$.

Lemma 5.9. The extended period map $\Phi^{H}: \mathcal{T}^{H} \rightarrow D$ still satisfies the Griffiths transversality.

Proof. Let us consider the tangent bundles $T^{1,0} \mathcal{T}^{H}$ and $T^{1,0} D$ as two differential manifolds, and the tangent map $\left(\Phi^{H}\right)_{*}: T^{1,0} \mathcal{T}^{H} \rightarrow T^{1,0} D$ as a continuous map. We only need to show that the image of $\left(\Phi^{H}\right)_{*}$ is contained in the horizontal tangent bundle $T_{h}^{1,0} D$.

The horizontal subbundle $T_{h}^{1,0} D$ is a close set in $T^{1,0} D$, so the preimage of $T_{h}^{1,0} D$ under $\left(\Phi^{H}\right)_{*}$ is a close set in $T^{1,0} \mathcal{T}^{H}$. On the other hand, because the period map $\Phi$ satisfies the Griffiths transversality, the image of $\Phi_{*}$ is in the horizontal subbundle 
$T_{h}^{1,0} D$. This means that the preimage of $T_{h}^{1,0} D$ under $\left(\Phi^{H}\right)_{*}$ contains both $T^{1,0} \mathcal{T}$ and the closure of $T^{1,0} \mathcal{T}$, which is $T^{1,0} \mathcal{T}^{H}$. This finishes the proof.

Proof of Theorem 5.6. By Theorem 5.3, we can see that $\mathcal{T}^{H}$ is a bounded domain in $\mathbb{C}^{N}$. Therefore, once we show $\mathcal{T}^{H}$ is domain of holomorphy, the existence of KählerEinstein metric on it follows directly from the well-known theorem by Mok-Yau in [11].

In order to show that $\mathcal{T}^{H}$ is a domain of holomorphy in $\mathbb{C}^{N}$, it is enough to construct a plurisubharmonic exhaustion function on $\mathcal{T}^{H}$.

Let $f$ be the exhaustion function on $D$ constructed in Proposition 5.8, whose Levi form, when restricted to the horizontal tangent bundle $T_{h}^{1,0} D$ of $D$, is positive definite at each point of $D$. We claim that the composition function $f \circ \Phi^{H}$ is the demanded plurisubharmonic exhaustion function on $\mathcal{T}^{H}$.

By the Griffiths transversality of $\Phi^{H}$, the composition function $f \circ \Phi^{H}: \mathcal{T}^{H} \rightarrow \mathbb{R}$ is a plurisubharmonic function on $\mathcal{T}^{H}$. Thus it suffices to show that the function $f \circ \Phi^{H}$ is an exhaustion function on $\mathcal{T}^{H}$, which is enough to show that for any constant $c \in \mathbb{R}$, $\left(f \circ \Phi^{H}\right)^{-1}(-\infty, c]=\left(\Phi^{H}\right)^{-1}\left(f^{-1}(-\infty, c]\right)$ is a compact set in $\mathcal{T}^{H}$. Indeed, it has already been shown in [5] that the set $f^{-1}(-\infty, c]$ is a compact set in $D$. Now for any sequence $\left\{p_{k}\right\}_{k=1}^{\infty} \subseteq\left(f \circ \Phi^{H}\right)^{-1}(-\infty, c]$, we have $\left\{\Phi^{H}\left(p_{k}\right)\right\}_{k=1}^{\infty} \subseteq f^{-1}(-\infty, c]$. Since $f^{-1}(-\infty, c]$ is compact in $D$, the sequence $\left\{\Phi^{H}\left(p_{k}\right)\right\}_{k=1}^{\infty}$ has a convergent subsequence. We denote this convergent subsequence by $\left\{\Phi^{H}\left(p_{k_{n}}\right)\right\}_{n=1}^{\infty} \subseteq\left\{\Phi^{H}\left(p_{k}\right)\right\}_{k=1}^{\infty}$ with $k_{n}<$ $k_{n+1}$, and denote $\lim _{k \rightarrow \infty} \Phi^{H}\left(p_{k}\right)=o_{\infty} \in D$. On the other hand, since the map $\Phi^{H}$ is injective and the Hodge metric on $\mathcal{T}^{H}$ is induced from the Hodge metric on $D$, the extended period map $\Phi^{H}$ is actually a global isometry onto its image. Therefore the sequence $\left\{p_{k_{n}}\right\}_{n=1}^{\infty}$ is also a Cauchy sequence that converges to $\left(\Phi^{H}\right)^{-1}\left(o_{\infty}\right)$ with respect to the Hodge metric in $\left(f \circ \Phi^{H}\right)^{-1}(-\infty, c] \subseteq \mathcal{T}^{H}$. In this way, we have proved that any sequence in $\left(f \circ \Phi^{H}\right)^{-1}(-\infty, c]$ has a convergent subsequence. Therefore $\left(f \circ \Phi^{H}\right)^{-1}(-\infty, c]$ is compact in $\mathcal{T}^{H}$, as was needed to show.

\section{REFERENCES}

[1] X. Chen, F. Guan, And K. Liu, Hodge metric completion of Teichmüller space of Calabi-Yau manifolds, arXiv:1305.0231.

[2] X. Chen, F. Guan And K. Liu, Global Torelli theorem for projective Manifolds of Calabi-Yau type, arXiv: 1205.4207

[3] F. ForSTNERIč, Stein manifolds and holomorphic mappings: the homotopy principle in complex analysis, Ergebnisse der Mathematik und ihrer Grenzgebiete 3, 56, Springer-Verlag, Berlin-Heidelberg, (2011).

[4] P. Griffiths, Periods of integrals on algebraic manifolds I, II, Amer. J. Math., 90 (1968), pp. 568-626, pp. 805-865.

[5] P. Griffiths And W. Schmid, Locally homogeneous complex manifolds, Acta Math., 123 (1969), pp. 253-302.

[6] Harish-Chandra, Representation of semisimple Lie groups VI, Amer. J. Math., 78 (1956), pp. $564-628$.

[7] S. Helgason, Differential geometry, Lie groups, and symmetric spaces, Academic Press, New York, (1978).

[8] L. HöRmANDER, An introduction to complex analysis in several variables, Van Nostrand, Princeton, NJ, (1973).

[9] P. W. Michor, Topics in differential geometry, Amer. Math. Soc., Providence, RI, (2008)

[10] N. Mok, Metric Rigidity Theorems on Hermitian Locally Symmetric Manifolds, Ser. Pure Math. Vol.6, World Scientific, Singapore-New Jersey-London-HongKong, (1989).

[11] N. Mok And S. T. YAU, Completeness of the Kähler-Einstein metric on bounded domains and the characterization of domain of holomorphi by curvature condition, The mathematical heritage of Henri Poincaré, Part 1 (Bloomington, Ind., 1980), Sympo. in Pure Math., 39 (1983), American Mathematics Society, Providence, Rhode Island, pp. 41-60. 
[12] S. Kobayashi And K. Nomizu, Foundations of differential geometry I, Wiley-Interscience, New York-London, (1963).

[13] K. Kodaira And J. Morrow, Complex manifolds, AMS Chelsea Publishing, Porvindence, RI, (2006), Reprint of the 1971 edition with errata.

[14] Y. Matsushima, Affine structure on complex manifold, Osaka J. Math., 5 (1968), pp. 215-222.

[15] H. Popp, Moduli theory and classification theory of algebraic varieties, Volume 620 of Lecture Notes in Mathemathics, Springer-Verlag, Berlin-New York, (1977).

[16] W. Schmid, Variation of Hodge structure: the singularities of the period mapping, Invent. Math., 22 (1973), pp. 211-319.

[17] Y. Shimizu and K. Ueno, Advances in moduli theory, Volume 206 of Translation of Mathematical Monographs, American Mathematics Society, Providence, Rhode Island, (2002).

[18] M. Sugiura, Conjugate classes of Cartan subalgebra in real semi-simple Lie algebras, J. Math. Soc. Japan, 11 (1959), pp. 374-434.

[19] M. Sugiura, Correction to my paper: Conjugate classes of Cartan subalgebra in real semisimple Lie algebras J. Math. Soc. Japan, 23 (1971), pp. 374-383.

[20] B. SzendröI, Some finiteness results for Calabi-Yau threefolds, Journal of the London Mathematical Society, Second series, 60 (1999), pp. 689-699.

[21] G. Tian, Smoothness of the universal deformation space of compact Calabi-Yau manifolds and its Petersson-Weil metric, Mathematical aspects of string theory(San Diego, Calif.), (1986), World Sci. Publishing, Singapore, Adv. Ser. Math. Phys., 1 (1987), pp. 629-646.

[22] A. N. Todorov, The Weil-Petersson geometry of the moduli space of $\mathrm{SU}(n \geq 3)$ (Calabi-Yau) manifolds. I, Comm. Math. Phys., 126 (1989), pp. 325-346.

[23] E. VIEHWEG, Quasi-projective moduli for polarized manifolds, Volume 30 of "Ergebnisse der Mathematik und ihrer Grenzgebiete (3) [Results in Mathematics and Related Areas (3)]", Springer-Verlag, Berlin, (1995).

[24] C. Voisin, Hodge theory and complex algebraic geometry I, Cambridge Universigy Press, New York, (2002).

[25] Y. Xu, Lie groups and Hermitian symmetric spaces, Science Press in China, (2001). (Chinese) 
